\title{
Visco-plastic models of isothermal lava domes
}

\author{
By N. J. BALMFORTH ${ }^{1}$, A. S. BURBIDGE ${ }^{2}$, \\ R. V. CRASTER ${ }^{3}$, J. SALZIG AND A. SHEN \\ ${ }^{1}$ Instituto di Cosmogeofisica, C. Fiume 4, 10133 Torino, Italy \\ ${ }^{2}$ School of Chemical Engineering, University of Birmingham, Edgbaston, \\ Birmingham, B15 2TT, UK \\ ${ }^{3}$ Department of Mathematics, Imperial College of Science, Technology and Medicine, \\ London, SW7 2BZ, UK \\ ${ }^{4}$ Woods Hole Oceanographic Institution, Woods Hole, MA 02543, USA \\ ${ }^{5}$ Department of Theoretical and Applied Mechanics, University of Illinois at Urbana-Champaign, \\ Urbana, Illinois 61801-2935, USA
}

(Received 25 January 1999 and in revised form 13 August 1999)

The dynamics of expanding domes of isothermal lava are studied by treating the lava as a viscoplastic material with the Herschel-Bulkley constitutive law. Thin-layer theory is developed for radially symmetric extrusions onto horizontal plates. This provides an evolution equation for the thickness of the fluid that can be used to model expanding isothermal lava domes. Numerical and analytical solutions are derived that explore the effects of yield stress, shear thinning and basal sliding on the dome evolution. The results are briefly compared with an experimental study. It is found that it is difficult to unravel the combined effects of shear thinning and yield stress; this may prove important to studies that attempt to infer yield stress from morphology of flowing lava.

\section{Introduction}

Both rheological experiments on lava (Shaw 1969; McBirney \& Murase 1984; Pinkerton \& Stevenson 1992) and the actual morphology of real lava flows (Hulme 1974; Blake 1990; Fink \& Griffiths 1998) suggest that, over a significant range of temperatures, silicic lava behaves like a visco-plastic fluid; that is, due to its crystal content, lava has a yield stress. In the simplest geometrical setting, namely the extrusion of such a lava from a small vent onto a horizontal surface, this yield stress is thought to shore up thick domes against gravity and support vertical 'spines' and upheaval plugs. From a practical viewpoint, these domes can be the setting of violent and dangerous events: domes can rupture and release confined gas in a pyroclastic outflow, or collapse at their periphery to create landslides and debris flows. Two views of a dome that grew inside the crater of Mount St Helens are shown in figure 1. Notably, these domes are nearly axisymmetric, of relatively low aspect ratio and evolved slowly, features which all suggest that some progress may be possible using mathematical modelling.

In addition to the yield stress, lavas also have at least two other non-Newtonian qualities: a strongly temperature-dependent viscosity, and solidification. It is upon these second two effects that most previous work has concentrated, and it is only 

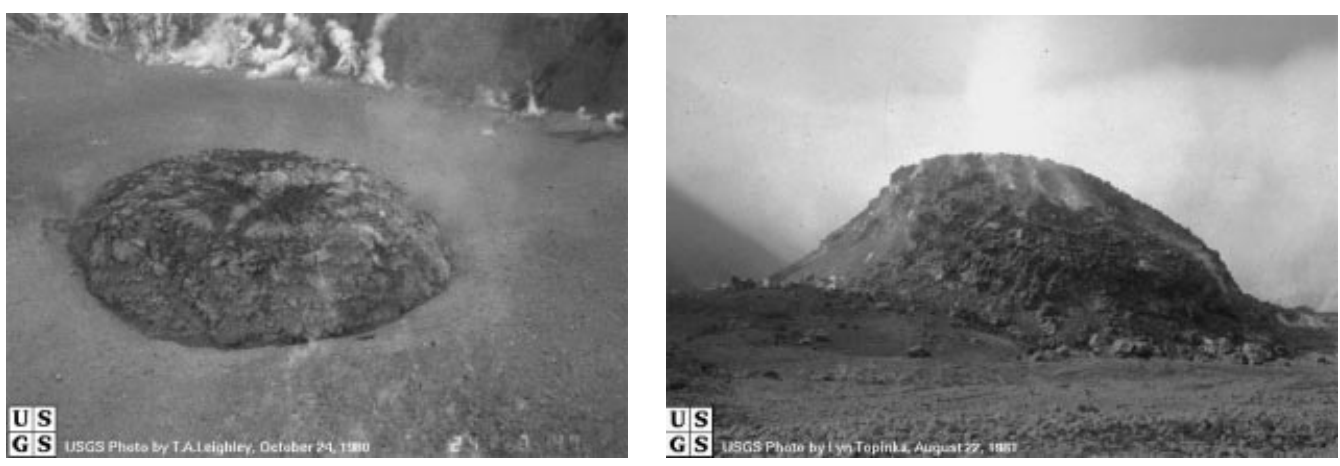

Figure 1. Two views of a lava dome on Mount St Helens that started growing on October 18 1980. When photographed for the top view (October 24, 1980) the dome was $34 \mathrm{~m}$ high and 300 $\mathrm{m}$ wide. The side view shows the dome in August 1981, when it was $163 \mathrm{~m}$ high and $400 \mathrm{~m}$ wide. Photographs are courtesy of the USGS/Cascades Volcano Observatory and further details regarding these domes and others can be found at http://vulcan.wr.usgs.gov/home.html

lately that attention has focused on the lava yield stress. For non-isothermal flows, the viscosity variation and the formation of a solidified crust are undoubtedly important. But if the lava does not cool significantly as it flows, the yield stress may play a dominant role. It is to this latter situation that we direct our attention is this study.

One way to study lava flow is through laboratory experiments with analogue materials. Such materials include viscous fluids (Huppert 1982), water-clay (kaolin) slurries (Hulme 1974; Blake 1990), cooling wax (Hallworth, Huppert \& Sparks 1987; Fink \& Griffiths 1992) or corn syrup (Stasiuk, Jaupart \& Sparks 1993), and mixtures of clay and wax (Griffiths \& Fink 1997). Clay suspensions typically impart a yield stress to the fluid, whereas wax and corn syrup have temperature-dependent viscosity. Wax also solidifies if the experiment is conducted at the right temperature, and so, in principle, wax-kaolin slurries potentially possess the three significant non-Newtonian effects present in lava.

But for isothermal studies of yield stress effects, water-clay slurries are most useful since they are standard materials used in chemical engineering and have welldocumented rheological properties. The extrusion of such a slurry onto a horizontal plane then provides the experimental analogue of a slowly cooling lava dome. One such experimental extrusion is shown in figure 2; this picture displays a snapshot of an expanding dome of kaolin-water slurry in air. Figures 1 and 2 are essentially the motivating images for the present study. Some physical properties of these slurries are shown in tables 1 and 2 .

Experimental studies have elucidated several features of lava flow dynamics. In particular, by comparing the morphology of laboratory analogues with those of real lava flows, the occurrence of certain naturally occurring features has been rationalized. But several studies have attempted to advance further and infer the rheology of real lava, based on this comparison. In particular, by extruding visco-plastic fluids such as clay-water slurries, and recording the shape of the expanding dome (such as that in figure 2), there have been several attempts to infer lava yield stress (Hulme 1974; Blake 1990).

On the theoretical modelling side, significantly less has been accomplished. Theory to date mostly consists of a set of scaling arguments for cooling flows (Griffiths \& Fink 1993), though Huppert (1982) gave similarity solutions suitable for radial extrusions of isothermal viscous fluid and Nye (1952) presented a solution for the 


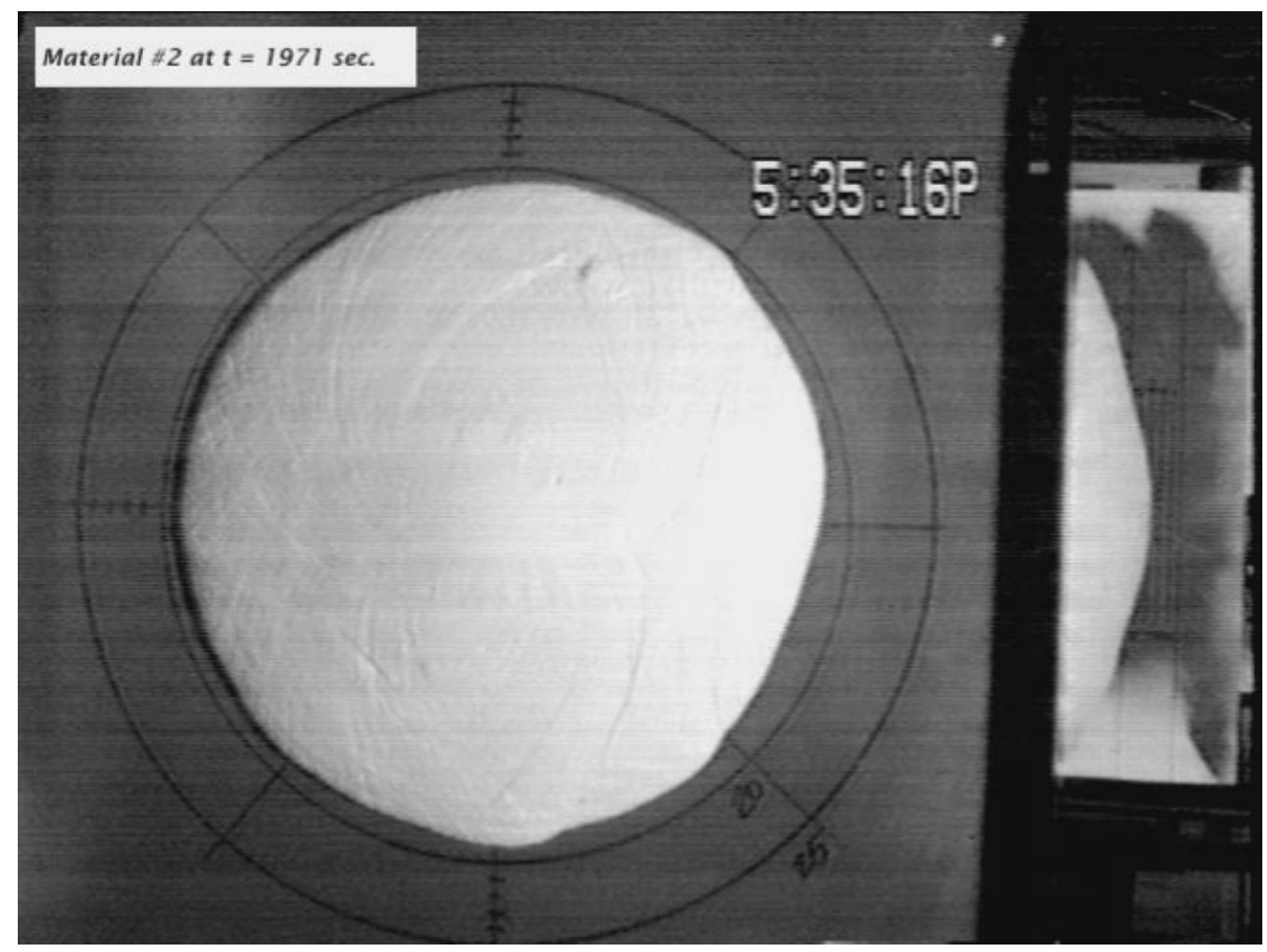

FIGURE 2. Snapshot of top and side view of a growing dome created by extruding a slurry of kaolin and water onto a horizontal, smooth aluminium plate in air. Pictures are taken using a CCD camera placed $1.5 \mathrm{~m}$ above the plate; a mirror placed at a $45^{\circ}$ angle to the edge of the plate enables both side and top views to appear in the same frame. The slurry is $1.2: 1$, kaolin to water, by weight.

radial flow of plastic (large yield stress) material. However, visco-plastic fluid models are also used in the theory of the flow of glaciers (Hutter 1983) and muds (Liu \& Mei 1989, 1991; Coussot 1997), and have been used to describe debris flows (Davies 1986) and snow avalanches (Dent \& Lang 1983). By contrast, mathematical modelling in these theories is significantly more advanced. The purpose of the present work is to outline some related theoretical developments for lava flow.

The simplest visco-plastic fluid is described by the Bingham model. This model has the characteristic relationship between stress and strain rate shown in figure 3: beyond a certain yield stress, the stress increases linearly with strain rate. A more versatile non-Newtonian fluid model is the Herschel-Bulkley fluid. This model has a power-law relationship between stress and strain rate once the yield stress is exceeded (figure 3), and compares more favourably with rheological measurements of clay slurries and muds (Coussot 1994, 1997; Huang \& Garcia 1998). Indeed the stressstrain-rate curves shown in Blake (1990), Hulme (1974), Griffiths \& Fink (1997) all clearly have a nonlinear dependence upon the strain rate. We illustrate this feature further in figure $3(b)$, which shows the relationship between the stress and strain rate for the mixture shown as an expanding dome in figure 2. To allow greater flexibility we therefore adopt the Herschel-Bulkley constitutive model.

In this paper we consider isothermal fluid flows, and so the problem does not contain the mathematical and physical complications associated with temperature- 

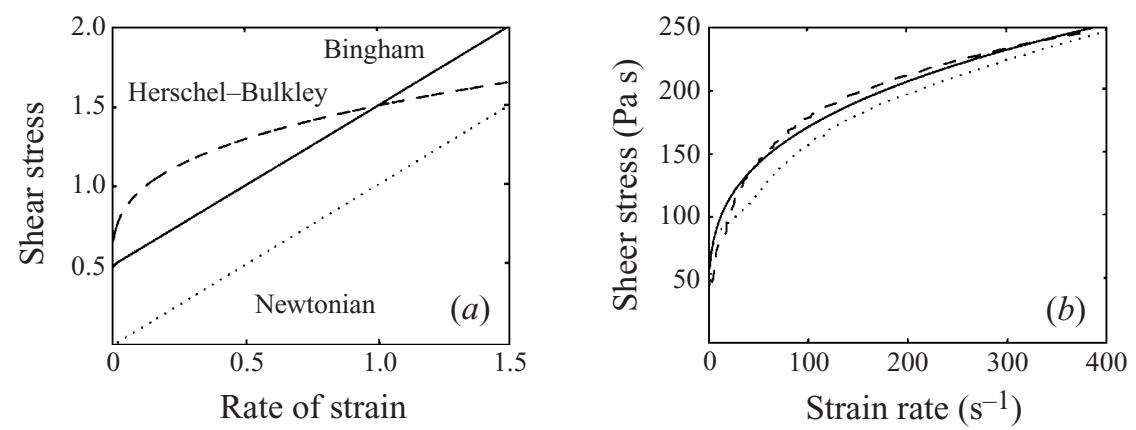

FIGURE 3. Non-Newtonian fluid models. (a) A sketch of the constitutive models for the Bingham fluid (solid line), a Newtonian fluid (dotted line) and a Herschel-Bulkley material (dashed curve); the scales are arbitrary in this panel. (b) Real rheological data for the mixture used in figure 2 (which is $1.2: 1$, kaolin to water, by weight). The measurements were made on a TA Instruments CSL500 controlled stress rheometer with stress first increasing, then decreasing. The dotted lines are measurements for increasing stress, and the dashed lines are for decreasing stress. The curves are slightly different and show some hysteresis; this is typical of a rheopectic material (a material with strain hardening structure development), and shows that the slurry is even more complicated in its rheology than we admit here. A fitted Herschel-Bulkley model is shown as the solid line. In both $(a)$ and $(b)$, the index of the Herschel-Bulkley model is $n=1 / 3$.

dependent rheology and solidification; we aim to begin a theoretical discussion of the thermal effects in a sequel. The major non-Newtonian effect that we model is the yield stress. More specifically, we exploit a thin-layer theory to derive a reduced model of the flow dynamics. This reduced model consists of an evolution equation for the local thickness of the fluid layer and is similar to equations derived for spreading mud (Liu \& Mei 1991). The mathematical formulation of the isothermal problem is described in $\S 2$. The expansion that leads to the evolution equation follows in $\S 3$. The next three sections detail various solutions to our equation, both analytical and numerical. In $\S 7$, we generalize the theory to account for the possibility that the visco-plastic fluid slips over the surface onto which it is extruded. The potential importance of this physical addition is suggested by rheological measurements of clays and slurries (Barnes 1995), and by Griffiths \& Fink (1997) for isothermal dome extrusions below water. In $\S 8$, we compare the predictions of the theory with a laboratory experiment in which a clay-water slurry is extruded onto a horizontal plate (figure 2). Section 9 is our point of summary. A preliminary report by Shen (1998) describes both isothermal and non-isothermal extrusions.

\section{Formulation}

\subsection{The equations}

We consider a thin film of incompressible fluid on a flat plane with axisymmetry. The fluid is described by the velocity field, $(u(r, z, t), 0, w(r, z, t))$, density, $\rho$, and pressure, $p(r, z, t)$. The equations for the film are

$$
\begin{gathered}
u_{t}+u u_{r}+w u_{z}=-\frac{1}{\rho} p_{r}+\frac{1}{\rho}\left[\partial_{r} \tau_{r r}+\partial_{z} \tau_{r z}+\frac{1}{r}\left(\tau_{r r}-\tau_{\theta \theta}\right)\right], \\
w_{t}+u w_{r}+w w_{z}=-\frac{1}{\rho} p_{z}-g+\frac{1}{\rho}\left[\partial_{r} \tau_{z r}+\partial_{z} \tau_{z z}+\frac{\tau_{r z}}{r}\right]
\end{gathered}
$$


and

$$
\frac{1}{r} \partial_{r}(r u)+w_{z}=0,
$$

where the $\tau_{i j}$ denote the deviatoric stresses and the symbol $\partial_{r}$ denotes partial differential with respect to $r$ and so forth. For a visco-plastic fluid, these stresses are related to the strain rates through a constitutive model; this will be discussed shortly.

We solve these equations subject to the free-surface conditions

$$
h_{t}+u h_{r}=w
$$

and

on $z=h(r, t)$.

$$
\left(\begin{array}{ccc}
\tau_{r r}-p & 0 & \tau_{r z} \\
0 & \tau_{\theta \theta}-p & 0 \\
\tau_{z r} & 0 & \tau_{z z}-p
\end{array}\right)\left(\begin{array}{c}
-h_{r} \\
0 \\
1
\end{array}\right)=\left(\begin{array}{l}
0 \\
0 \\
0
\end{array}\right)
$$

At the base we impose no slip on the velocity field, except just above the vent where there is a source of fluid with a specified vertical velocity (we ignore the back-reaction of the dome upon the fluid moving up the vent):

$$
u=0 \quad \text { and } \quad w=w_{s}(r, t) \quad \text { on } z=0 .
$$

This boundary condition is replaced by a slip law in $\S 7$ to study the effect of basal sliding. In all illustrative examples, the source term, $w_{s}(r, t)$ is taken to be of the form

$$
w_{s}(r, t)=w_{0}(t)\left(r_{*}^{2}-r^{2}\right) \vartheta\left(r_{*}^{2}-r^{2}\right),
$$

where $w_{0}$ depends only on $t, r_{*}$ is the vent radius and $\vartheta(x)$ indicates the step function.

\subsection{Constitutive model}

As we discussed in the introduction, the constitutive relation we employ is the Herschel-Bulkley model,

$$
\tau_{i j}=\left(K \dot{\gamma}^{n-1}+\frac{\tau_{p}}{\dot{\gamma}}\right) \dot{\gamma}_{i j} \quad \text { for } \tau \geqslant \tau_{p}
$$

and

$$
\dot{\gamma}_{i j}=0 \quad \text { for } \tau<\tau_{p}
$$

(Oldroyd 1947; Herschel \& Bulkley 1923), where $\dot{\gamma}_{i j}$ is defined here as

$$
\dot{\gamma}_{i j}=\left(\begin{array}{ccc}
2 u_{r} & 0 & u_{z}+w_{r} \\
0 & 2 u / r & 0 \\
u_{z}+w_{r} & 0 & 2 w_{z}
\end{array}\right),
$$

that is, it is twice the rate-of-strain tensor. The second invariants of $\tau_{i j}$ and $\dot{\gamma}_{i j}$ are defined as

$$
\tau=\sqrt{\frac{1}{2} \tau_{j k} \tau_{j k}} \quad \text { and } \quad \dot{\gamma}=\sqrt{\frac{1}{2} \dot{\gamma}_{j k} \dot{\gamma}_{j k}}
$$

The yield stress is $\tau_{p}$, and $K$ is the consistency. For a Bingham material, once the yield stress is exceeded, the deviatoric stresses are directly proportional to the shear rate; thus $n=1$ and $K=\eta=\rho v$, where $\eta$ is the plastic viscosity and $v$ is the kinematic viscosity. Hence, in this case, $K$ is just conventional viscosity. In general, $K$ provides a measure of resistance to shear. The parameter $n$ characterizing the nonlinearity in the flow regime determines whether the fluid is shear thinning $(n<1)$ or shear thickening $(n>1)$; lavas are almost invariably assumed to be shear thinning. However, this may 


\section{Constants}

Density, $\rho\left(\mathrm{kg} \mathrm{m}^{-3}\right)$

Viscosity, $\eta$ (Pa s)

Yield stress, $\tau_{p}(\mathrm{~Pa})$

Index, $n$,

$\begin{array}{cc}\text { Silicic lava } & \text { Wax-kaolin slurry } \\ 2600 & 1320 \\ 10^{9} & 15 \\ 10^{5} & 14 \\ 1 & 0.35\end{array}$

TABLE 1. Physical constants for lava and a kaolin-water slurry used in laboratory experiments (§8). The data for lava are taken from Griffiths \& Fink (1993), McBirney \& Murase (1984), Shaw (1969). The characteristic value of viscosity corresponds to the maximal value at the low end of the temperature range.

not be true for extremely high crystal concentrations (Smith 1997) which may occur close to the freezing temperature.

\subsection{Non-dimensionalization}

We non-dimensionalize the equations as follows: $H$, a characteristic thickness of the fluid layer, is taken as the dimension of $z$, and $L$ as a horizontal length scale. We measure the speeds, $u$ and $w$, by $V$ and $H V / L$ respectively, and time by $L / V$. Then,

$$
r=L \tilde{r}, \quad z=H \tilde{z}, \quad u=V \tilde{u}, \quad w=(V H / L) \tilde{w}, \quad t=(L / V) \tilde{t} \quad \text { and } \quad h=H \tilde{h} .
$$

For pressure we introduce

and for general $n$ we define

$$
p=\rho g H \tilde{p},
$$

$$
\eta=\frac{K V^{n-1}}{H^{n-1}}
$$

and then select $V=\rho g H^{3} / \eta L$. This definition of $V$ assumes balances between gravity and hydrostatic pressure, and between the horizontal pressure gradient and the stress due to the vertical shear of the radial outflow. Note that it is more natural to take this relation as defining $H$, given the rate of extrusion, in the circumstance that we allow a fixed inflow rate through the vent. We will consider this explicitly when we turn to a laboratory experiment in $\S 8$.

On substituting the non-dimensional variables into the governing equations, and dropping the tildes, we arrive at

$$
\epsilon^{2} \operatorname{Re}\left(u_{t}+u u_{r}+w u_{z}\right)=-p_{r}+\epsilon \partial_{r} \tau_{r r}+\partial_{z} \tau_{r z}+\frac{\epsilon}{r}\left(\tau_{r r}-\tau_{\theta \theta}\right)
$$

and

$$
\epsilon^{4} \operatorname{Re}\left(w_{t}+u w_{r}+w w_{z}\right)=-p_{z}-1+\epsilon^{2} \partial_{r} \tau_{z r}+\epsilon \partial_{z} \tau_{z z}+\epsilon^{2} \frac{\tau_{r z}}{r} .
$$

The stresses are given explicitly in (2.21), (2.22) below. The incompressibility condition remains as

$$
\frac{1}{r} \partial_{r}(r u)+w_{z}=0 .
$$

In the above equations, $\epsilon=H / L \ll 1$ is the small aspect ratio of the fluid layer.

We also define a Reynolds number as

$$
R e=\frac{V^{2}}{g H}\left(\frac{H^{2}}{L^{2}}\right)^{-1} \equiv \frac{\rho^{2} g H^{3}}{\eta^{2}} \equiv \frac{\rho V L}{\eta} .
$$




$\begin{array}{lcc}\text { Extrusion conditions } & \text { Lava } & \text { Kaolin-water } \\ \text { Dome radius, } L(\mathrm{~m}) & 10^{2} & 0.10 \\ \text { Effusion rate, } Q\left(\mathrm{~m}^{3} \mathrm{~s}^{-1}\right) & 0.1-10 & 10^{-6}\end{array}$

TABLE 2. Typical extrusion conditions for lava and kaolin-water experiments. The data for lava is taken from Griffiths \& Fink (1993), McBirney \& Murase (1984) and Shaw (1969).

Non-dimensional parameter

$$
\begin{aligned}
& \epsilon=H / L \\
& R e=\rho V L / \eta \\
& B=\tau_{p^{*}} H / \eta V
\end{aligned}
$$

Lava

$\begin{array}{lc}\left(\eta Q / \rho g q L^{4}\right)^{1 / 4} & 0.1 \\ \left(\rho^{5} g Q^{3} / \eta^{5} q^{3}\right)^{1 / 4} & 10^{-8} \\ \tau_{p^{*}} L(q / \eta \rho g Q)^{1 / 2} & 1\end{array}$

Kaolin-water

0.01

1

100

TABLE 3. Typical values of non-dimensional numbers, given the physical constants and extrusion conditions in tables 1 and 2, assuming $n=1, q=\pi$ and $Q=1 \mathrm{~m}^{3} \mathrm{~s}^{-1}$ for lava. The parameter $q$ is a non-dimensional flow rate introduced in $\S 8$ where we relate the analysis to an experiment. (By taking $q \sim 1$, we scale the problem so that the extrusion time is order unity and no further rescalings of time are necessary; this is unlike our numerical computations reported in $\S 6$ in which $q \sim 10^{-4}$ and extrusion times are long.)

We take $R e$ to be order unity, which ensures a low Reynolds number flow, or, equivalently, the absence of inertial effects to leading orders (see table 3 for typical values).

Next, with the units $V / H$, the components of $\dot{\gamma}_{i j}$ become

$$
\dot{\gamma}_{\theta \theta}=2 \epsilon u / r, \quad \dot{\gamma}_{r r}=2 \epsilon u_{r}, \quad \dot{\gamma}_{r z}=u_{z}+\epsilon^{2} w_{r} \quad \text { and } \quad \dot{\gamma}_{z z}=2 \epsilon w_{z},
$$

and

$$
\dot{\gamma}=\left[\left(u_{z}+\epsilon^{2} w_{r}\right)^{2}+4 \epsilon^{2} u_{r}^{2}+4 \epsilon^{2}(u / r)^{2}\right]^{1 / 2} .
$$

Hence, in the units $\rho g H^{2} / L$ and provided $\tau>B$, the dimensionless stresses are given by

$$
\tau_{r r}=2 \epsilon\left(\dot{\gamma}^{n-1}+\frac{B}{\dot{\gamma}}\right) u_{r}, \quad \tau_{z z}=2 \epsilon\left(\dot{\gamma}^{n-1}+\frac{B}{\dot{\gamma}}\right) w_{z}
$$

and

$$
\tau_{\theta \theta}=2 \epsilon\left(\dot{\gamma}^{n-1}+\frac{B}{\dot{\gamma}}\right) u / r, \quad \tau_{r z}=\left(\dot{\gamma}^{n-1}+\frac{B}{\dot{\gamma}}\right)\left(u_{z}+\epsilon^{2} w_{r}\right),
$$

with

$$
\tau=\sqrt{\tau_{r z}^{2}+\frac{1}{2}\left(\tau_{r r}^{2}+\tau_{\theta \theta}^{2}+\tau_{z z}^{2}\right)} .
$$

The non-dimensional group,

$$
B=\frac{\tau_{p} H}{\eta V} \equiv \frac{\tau_{p} L}{\rho g H^{2}},
$$

is a measure of the yield stress relative to the horizontal pressure gradient acting over the thickness of the fluid; henceforth we refer to $B$ as the Bingham number, even if $n \neq 1$.

If $\tau<B$, on the other hand, then $u_{r}=u_{z}+\epsilon^{2} w_{r}=w_{z}=0$.

The rescaled boundary conditions are

$$
u=0 \quad \text { and } \quad w=w_{0}(t) f(r) \quad \text { on } z=0,
$$


and, on $z=h(r, t)$,

$$
h_{t}+u h_{r}=w
$$

and

$$
\tau_{r z}+p h_{r}=\epsilon h_{r} \tau_{r r} \quad \text { and } \quad p=\epsilon\left(\tau_{z z}-\epsilon h_{r} \tau_{r z}\right) .
$$

\section{The expansion}

We now solve the equations by introducing an asymptotic expansion. Though there are some subtleties in the expansion (Balmforth \& Craster 1999), we actually need only the leading-order terms of all the equations. These are

$$
\begin{gathered}
-p_{r}+\partial_{z} \tau_{r z}=0, \\
p_{z}=-1, \\
\frac{1}{r} \partial_{r}(r u)+w_{z}=0,
\end{gathered}
$$

and

subject to

$$
\dot{\gamma}=\left|u_{z}\right|,
$$

$$
u=0 \quad \text { and } \quad w=w_{0} f(r) \quad \text { on } z=0
$$

and

$$
h_{t}+u h_{r}=w \quad \text { and } \quad \tau_{r z}=p=0 \quad \text { on } z=h(r, t) .
$$

The constitutive model is

$$
\tau_{r z}=\left(\dot{\gamma}^{n-1}+\frac{B}{\dot{\gamma}}\right) u_{z}, \quad \text { for } \tau>B \equiv\left|\tau_{r z}\right|,
$$

and $u_{z}=0$ if $\tau<B$.

Equation (3.2) integrates to

$$
p=h(r, t)-z,
$$

and then (3.1) to

$$
\tau_{r z}=-h_{r}(h-z) .
$$

Evidently, $\tau=\left|h_{r}\right|(h-z)$ decreases with $z$ and the largest shear stress occurs along the base of the fluid. Here,

$$
\tau(r, 0, t)=\left|\tau_{r z}(r, 0, t)\right|=\left|h h_{r}\right|,
$$

which must exceed $B$ in order for the fluid to move at all. Furthermore, at $z=h$, $\tau=0$. Hence, if the fluid is moving, the stress must fall beneath the yield stress at some level, $z=Y(r, t)$. This surface is given by

$$
Y(r, t)=h-\frac{B}{\left|h_{r}\right|},
$$

with $Y>0$ in order for the fluid to move.

Below $z=Y$, the fluid is yielding and so

$$
B+\left|u_{z}\right|^{n-1} u_{z}=-h_{r}(h-z),
$$

or

$$
u_{z}=\left[\left|h_{r}\right|(Y-z)\right]^{1 / n} \operatorname{sgn}\left(-h_{r}\right) .
$$


Above $z=Y$ the fluid is rigid and $u_{z}=0$. That is, we have a 'plug flow'. The identification of the plug is actually a little confusing (Lipscomb \& Denn 1984) and some clarification is required; a more detailed discussion follows equation (3.17).

Now, we define

$$
U(r, t)=\int_{0}^{h} u(r, z, t) \mathrm{d} z \equiv \int_{0}^{h}(h-z) u_{z}(r, z, t) \mathrm{d} z .
$$

Then,

$$
U(r, t)=-\frac{n}{n+1}\left|h_{r}\right|^{1 / n-1} Y^{1+1 / n}\left(h-\frac{n Y}{2 n+1}\right) h_{r} \vartheta(Y),
$$

where $\vartheta(x)$ is the Heaviside step function. The introduction of this discontinuous function into $U(r, t)$ takes care of the yield criterion, which is that, if $Y<0$, the fluid is insufficiently stressed to move. In that case, the fluid as a whole is stationary and the free surface cannot evolve (we distinguish stationary from plug flow).

The quantity $U(r, t)$ is needed for the evolution equation for $h(r, t)$, which we now derive by vertically integrating the continuity equation (3.3) and using the free-surface condition in (3.6):

$$
h_{t}+\frac{1}{r} \partial_{r}(r U)=w_{s}
$$

The evolution equation (3.16) has a simple conservation law,

$$
\frac{\mathrm{d}}{\mathrm{d} t} \int_{0}^{\infty} h(r, t) r \mathrm{~d} r=\int_{0}^{\infty} w_{s}(r, t) r \mathrm{~d} r
$$

(conservation of mass), provided the only source of fluid is the vent at the origin.

Note that the evolution equation models a radially expanding flow. This means that the flow is extensional everywhere, and so there cannot be a true plug flow anywhere. This is seemingly inconsistent with the claim above that the region $h>z>Y$ contains a rigid plug (cf. Lipscomb \& Denn 1984). In fact, what really happens is that the leading-order solution breaks down inside this region. Further developments of the problem (Balmforth \& Craster 1999; see also Lawrence \& Corfield 1998) reveal that this region is, in fact, weakly yielding and $u_{z} \sim O(\epsilon)$. This slight yielding is created by the conspiracy of the order- $\epsilon$ stress components (which includes the normal stresses), and is sufficient to compensate the radial expansion. Importantly, the theory remains consistent. Another route out of this apparent inconsistency is to use a fluid model that does not have a real yield stress, but behaves as a very viscous fluid for small strain rates. This type of fluid model is sometimes claimed to be more realistic than Bingham-type models (Barnes \& Walters 1985), but this view is not uniformly accepted (N'Guyen \& Boger 1992). Although unnecessary for analytical work this alternative route is convenient for numerical reasons, and we outline some developments of the problem along this line in the Appendix; there we formulate the thin-layer theory for a modified constitutive model which yields weakly at small rates of strain. The purpose is to derive another version of our theory in which the non-smooth functions in (3.16) become smooth.

\section{Two limits in yield strength}

Two limiting cases of $B$ are of particular importance: The power-law fluid, $B \rightarrow 0$, and a high yield stress fluid, $B \rightarrow \infty$. We consider these for a constant source $\left(w_{s}=w_{s}(r)\right)$. 
4.1. Power-law fluid: $B \rightarrow 0$

When $B \rightarrow 0$, the effect of the yield stress vanishes. If $n=1$, we then recover the thin-film equation for Newtonian fluid (Huppert 1982):

$$
B=0, n=1: \quad h_{t}-\frac{1}{3 r} \partial_{r}\left(r h^{3} h_{r}\right)=w_{s} .
$$

Provided $w_{s}$ is a constant point source, this equation has a similarity solution,

$$
h(r, t)=f(r / \sqrt{t}) \equiv f(\eta),
$$

with

$$
-\frac{1}{2} \eta^{2} f_{\eta}=\frac{1}{3} \frac{\mathrm{d}}{\mathrm{d} \eta}\left(\eta f^{3} f_{\eta}\right)
$$

as found previously by Huppert (1982); see also Barenblatt (1979). Thus radius $R(t)$ and thickness $h(0, t)$ vary as $R \sim t^{1 / 2}$ and $h(0, t) \sim$ constant.

When $n$ is not unity, we obtain a thin-film equation for a power-law fluid:

$$
B=0, n \neq 1: \quad h_{t}-\frac{n}{(2 n+1) r} \partial_{r}\left(r h^{2+1 / n} h_{r}^{1 / n}\right)=w_{s} .
$$

(This is a version of the porous medium equation in radial geometry.) Again we have a similarity solution,

$$
h(r, t)=t^{\alpha} f\left(r / t^{\beta}\right)=t^{\alpha} f(\eta), \quad R(t)=R_{0} t^{\beta} \quad \text { and } \quad h(0, t)=h_{0} t^{\alpha},
$$

with

$$
\alpha=\frac{1-n}{3 n+5}, \quad \beta=2\left(\frac{1+n}{3 n+5}\right),
$$

and constants, $R_{0}$ and $h_{0}$.

\subsection{High yield stress: 'large' $B$}

The opposite limit, in which the fluid is dominated by the yield stress, and $B$ is in some sense large, is not so straightforward. As is evident from the numerical results presented later, this limit is characterized by solutions for which $Y \rightarrow 0$. That is, $h=\bar{h}+y$, where $|\bar{h}| \gg|y|$ for all time, and

$$
\bar{h}=-\frac{B}{\bar{h}_{r}}
$$

(so the associated yield surface is precisely zero). This solution, and the smallness of $y$ must be verified from the equation satisfied by $y$. From (4.7) we obtain

$$
\bar{h}= \begin{cases}\sqrt{2 B(R-r)}, & r<R \\ 0, & r>R\end{cases}
$$

where the time rate of change of the radius, $R(t)$, is dictated by the conservation law (3.17) and the time-dependence of the source. For a constant inflow rate, $Q$,

$$
R(t)=\frac{1}{(2 B)^{1 / 5}}\left(\frac{15 Q t}{8 \pi}\right)^{2 / 5}, \quad h(0, t)=(2 B R)^{1 / 2} .
$$

This solution represents a quasi-static expansion and a steady progression through the equilibrium solutions found by Nye (1952); see also Blake (1990).

The scalings in (4.6) and (4.9) can also be found from dimensional analysis (Griffiths $\&$ Fink 1993) in which one assumes a balance between terms representing the pressure 
gradient and basal shear stress in the governing equations. But these scalings only follow in the appropriate asymptotic limits, and for general parameter values there is no characteristic temporal dependence of height and radius of the form (4.5), as illustrated by the numerical results described shortly.

\section{A similarity solution for a time-dependent source}

If $w_{s}$ takes the form of a point source at $r=0$, then we may look for a similarity solution to the evolution equation (3.16) with the form

$$
h(r, t)=t^{\alpha} f\left(r / t^{\beta}\right)=t^{\alpha} f(\eta) .
$$

If $w_{s} \sim t^{\gamma}$, then the conservation law (3.17) implies that

$$
\alpha+2 \beta=\gamma .
$$

Moreover, direct substitution of (5.1) into the evolution equation gives

$$
\begin{aligned}
\frac{1}{n}(n+1)(2 n+1) \eta t^{\beta(1+1 / n)-\alpha(1+2 / n)-1}\left(\alpha f-\beta \eta f_{\eta}\right) & \\
= & \frac{\mathrm{d}}{\mathrm{d} \eta}\left[\left(f-\frac{n \mathscr{Y}}{2 n+1}\right) \mathscr{Y}^{1+1 / n}\left|f_{\eta}\right|^{1 / n-1} f_{\eta}\right],
\end{aligned}
$$

where the function $\mathscr{Y}$ is defined via

$$
Y=t^{\alpha}\left[f+\frac{B}{f_{\eta}} t^{\beta-2 \alpha}\right]_{+} \equiv t^{\alpha} \mathscr{Y},
$$

and where the + subscript is to remind us to take the quantity in brackets only where it is positive, and zero otherwise. Evidently, due to the form of $\mathscr{Y}$, a similarity solution will not in general exist. However, if $\beta<2 \alpha$, then ultimately $Y \rightarrow t^{\alpha} f(\eta)$ and the solution may converge to one of similarity form. In this case,

$$
\beta(1+n)=\alpha(2+n)+n \quad \text { and } \quad \beta<2 \alpha .
$$

The conditions (5.2) and (5.5) can be rewritten in the form

$$
\alpha=\frac{(n+1) \gamma-2 n}{3 n+5}, \quad \beta=\frac{n+\gamma(n+2)}{3 n+5}
$$

and $\gamma>5$.

There is a special case in which an exact similarity solution exists. This is when $\gamma=5$ and

$$
h(r, t)=t f\left(r / t^{2}\right)=t f(\eta)
$$

with

$$
\frac{1}{n}(n+1)(2 n+1) \eta\left(f-2 \eta f_{\eta}\right)=\frac{\mathrm{d}}{\mathrm{d} \eta}\left[\eta\left(f-\frac{n \mathscr{Y}}{2 n+1}\right) \mathscr{Y}^{1+1 / n}\left|f_{\eta}\right|^{1 / n-1} f_{\eta}\right]
$$

and $\mathscr{Y}=f+B / f_{\eta}$. This special case is significant also in Blake's (1990) dimensional analysis. In general, however, there is no solution of similarity form for a constant source $(\gamma=0)$. In that circumstance, the problematic term in $Y$ does not remain subdominant as it does if $\gamma>5$. 


\section{Numerical results}

We now describe some numerical results utilizing the thin-layer theory. The evolution equation

$$
h_{t}+\frac{1}{r} \partial_{r}(r U)=w_{s}
$$

together with equation (3.15) for $U(r, t)$, is solved using an adaptive grid scheme; the scheme utilized is designed to solve nonlinear parabolic equations and is described in some detail by Blom \& Zegeling (1994) $\dagger$ We also performed some comparative simulations using a finite element collocation scheme, PDECOL, Keast \& Muir (1991).

To avoid some numerical difficulties that occur where $h(r, t) \rightarrow 0$, we take an initial condition in which $h(r, t)$ is everywhere small and finite, $h(r, 0)=10^{-3} \exp \left(-r^{2} / 25\right)$, and add a linear viscous term to (6.1) with a very small coefficient $\left(10^{-8}\right)$. We also prescribe the gradient of $h$ at the origin, and at a radius $r_{\infty}$ that is sufficiently large to be considered infinite:

$$
h_{r}(0, t)=h_{r}\left(r_{\infty}, t\right)=0 .
$$

The only remaining term to identify is the source behaviour; for this we choose

$$
w_{s}(r)=0.1\left(r_{*}^{2}-r^{2}\right) \vartheta\left(r_{*}^{2}-r^{2}\right),
$$

and $r_{*}=0.15$, which prescribes the vent radius and flux. The value of 0.1 used for $w_{0}$ has no special significance, and, in fact, a suitable scaling of $r, t$ and $B$ can be used to explicitly remove this 'free parameter' from the problem.

Because our thin-layer equation contains some non-smooth functions, there are some minor numerical difficulties that are circumvented by smoothing out $U(r, t)$, as described in the Appendix. We have verified that the numerical solutions are insensitive to the details of this regularization and the size of the parameter $\mu$ which measures the degree of smoothing (see Appendix).

\subsection{Bingham fluids}

We first take $n=1$, which corresponds to the Bingham model, and explore the effect of varying the yield stress.

In figure 4 , we display results for a fluid with a very small yield stress $\left(B=10^{-5}\right)$ representative of an almost Newtonian fluid. This fluid behaves essentially like a Newtonian fluid, and converges to the similarity form $h(r, t)=f(r / \sqrt{t})$ away from the vent (see figure $4 c$ ). Because the source has finite radius, the similarity scaling is broken over the vent, $r<r_{*}$. Note that the extent of the source is reflected in the morphology of the dome.

A case with a relatively large yield stress is shown in figure 5; the value of $B$ here is 0.1 which is not actually a very large number; we enter the yield-stress-dominated limits at these low values of $B$ because of our choice of dimensional units and $w_{0}=0.1$ (see table 3 and $\S 8$ ). This value of $B$ is representative of a thick paste-like material. As we remarked earlier, $Y \rightarrow 0$ for the outflow. Consequently, the fluid marches slowly through an approximate sequence of equilibria given by Nye's parabolic profile (4.8). The radius and height then have the scalings (4.9), as illustrated by figure 5(c).

An intermediate case with moderate yield stress, $B=0.01$, is shown in figure 6; this is representative of a slurry which has a substantial proportion of water. Here the 'plug' occupies roughly half of the fluid (figure $6 a$ ), and it is not possible to collapse the solutions in figure 6 to one of similarity form.

$\dagger$ The code is available at the web-site: http://www.ma.ic.ac.uk/ rvcras/dome.f.gz. 

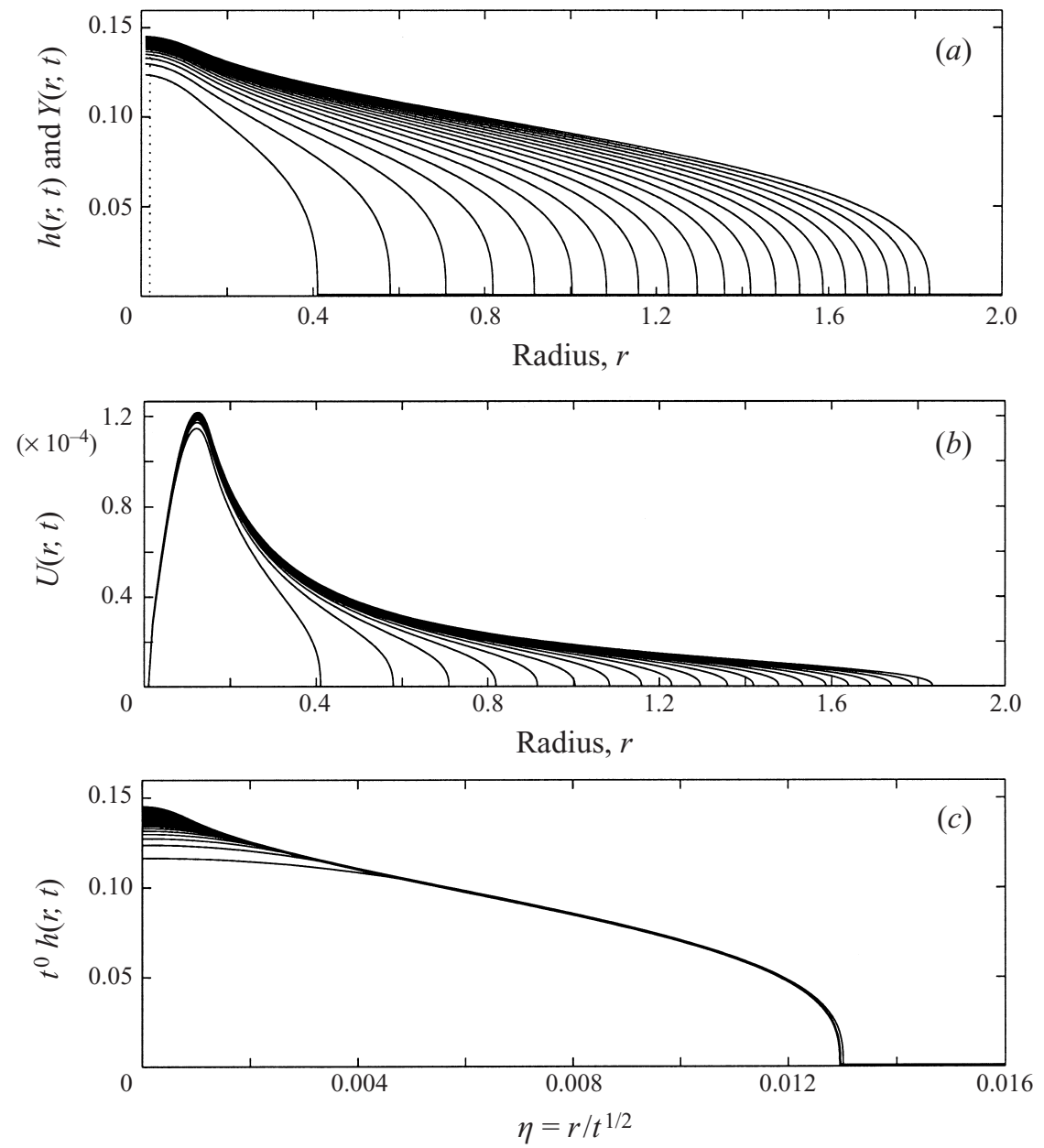

FIGURE 4. Evolution for $B=10^{-5}$. (a) The height field, $h(r, t)$, together with the 'yield' surface, $Y(r, t)$ shown by the dotted lines. The yield surface is everywhere indistinguishable from the height except in a slender region around the origin. $(b) U(r, t)$, the vertical integral of $u(r, z, t)$. (c) The height field is plotted against the similarity coordinate $\eta=r / t^{1 / 2}$. Shown are snapshots of the solution every 500 time units. The regularization parameter of the Appendix is $\mu=10^{-7}$.

A selection of dome shapes is shown in figure 7. This figure shows the height, $h(r, t)$, at $t=10^{4}$ for various computations using different $B$. As the yield stress increases, the aspect ratio of the dome increases and the shape becomes increasingly parabolic. $f$ Note that for the larger values of $B$, the dome appears to have a finite gradient at $r=0, h_{r}(0, t) \neq 0$, in violation of the symmetry condition imposed there. The reason for this is that if $h_{r} \rightarrow 0$, then $Y(0, t)=0$ and the central portion of the fluid never yields. But if $Y \rightarrow 0$, then the fluid at the centre of the dome cannot flow radially; instead we have the balance $h_{t}=w_{s}$, which implies a continual build-up of the dome centre. But such a fluid structure must yield sideways. The fluid finds its way out of this conundrum by building up the gradient of $h(r, t)$ in an increasingly narrow region around $r=0$. However, over the region with $r=O(\epsilon)$, the thin-layer theory breaks

$\mp$ The pictures are all displayed in dimensionless units. This means that $\epsilon h(0, t) / R(t)$ is the actual aspect ratio, which is much smaller (by the factor of $\epsilon$ ) than the figures appear to suggest. 

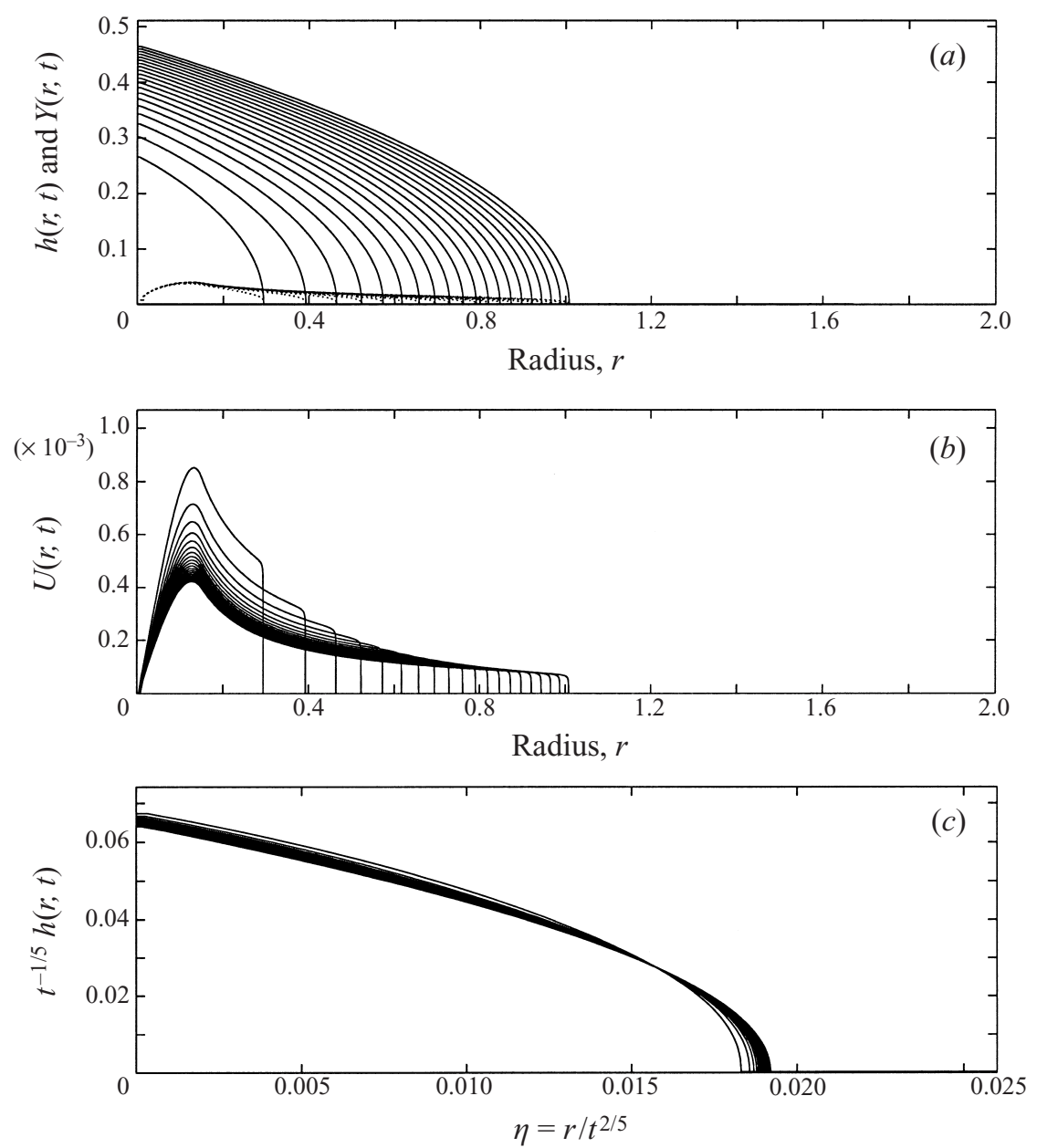

FIgURE 5 . As figure 4 but for $B=0.1$. In $(c)$, the height field is scaled by $t^{1 / 5}$ and plotted against the similarity coordinate $\eta=r / t^{2 / 5} \cdot \mu=10^{-4}$.

down and we should strictly use a different asymptotic expansion (cf. Johnson 1984). We assume that the global structure of the dome is insensitive to this slight deficiency in the model.

In figure 8 we show radius and height evolution for five values of $B$. (For computational convenience, we define the radius $R(t)$ as the location for which $h(R, t)=0.01$.) This shows the convergence to a scaling somewhere between the Newtonian and dominating yield-stress limits. One other feature brought out in this figure is the linear-in-time increase in the height field for small times. This stage of the dome growth involves the predominantly vertical rise of the fluid out of the vent: the fluid initially rises up like extruded toothpaste and does not yield radially. Eventually (and depending on the size of $B$ ), the extruded fluid yields under its own weight and flows radially (as shown in figure 9); similar behaviour has been noted in the flows of pastes (Burbidge \& Bridgwater 1999) and observed in the experiments of Griffiths \& Fink (1997). If the extrusion of fluid from the vent were abruptly to cease whilst the expansion was still in this phase, and solidification then to ensue, the dome would ap- 

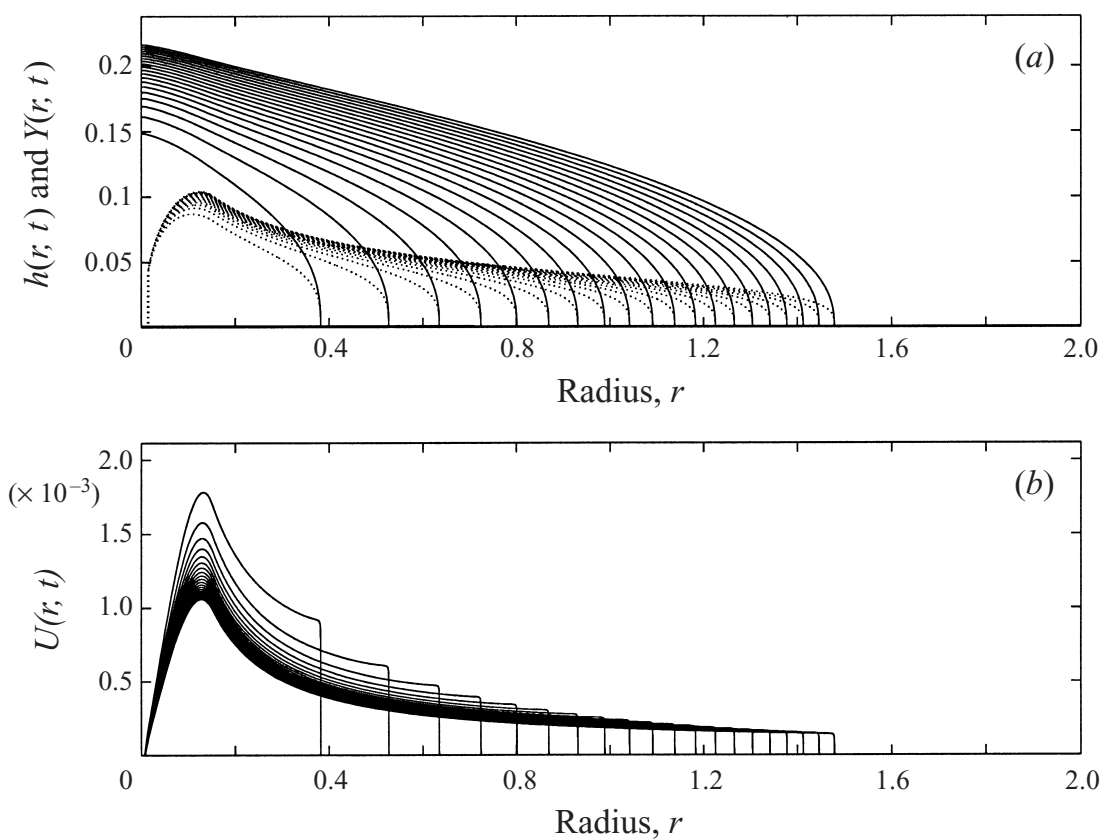

FiguRE 6. As figure $4(a, b)$ but for $B=0.01 . \mu=10^{-4}$.

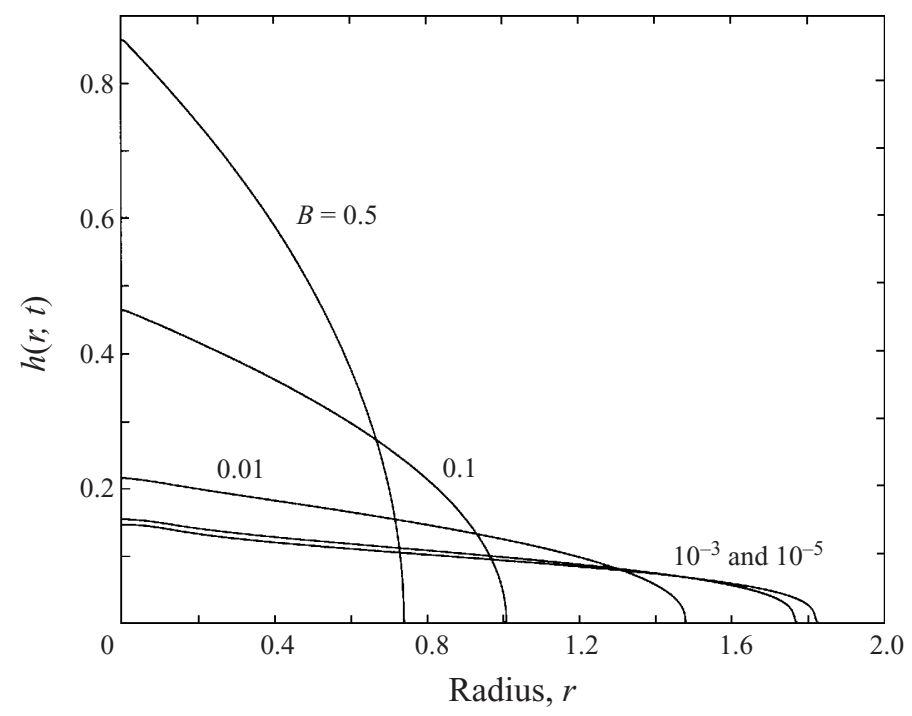

Figure 7. Dome shapes for varying $B$ after $t=10^{4} . \mu=10^{-2} B$.

pear more like a vertical column. This is a plausible mechanism for the emplacement of upheaval plugs.

An important property of the curves shown in figure 8 is that, after the fluid yields radially at $t \approx 10^{2}$, there are no abrupt transitions. In fact, $R(t)$ and $h(0, t)$ follow smooth curves over times much longer than those shown in figure $8(a-d)$; see figure $8(e)$ for $B=0.01$. This is contrary to expectations based on dimensional analysis (Blake 1990), which suggests that a transition occurs between viscous and 

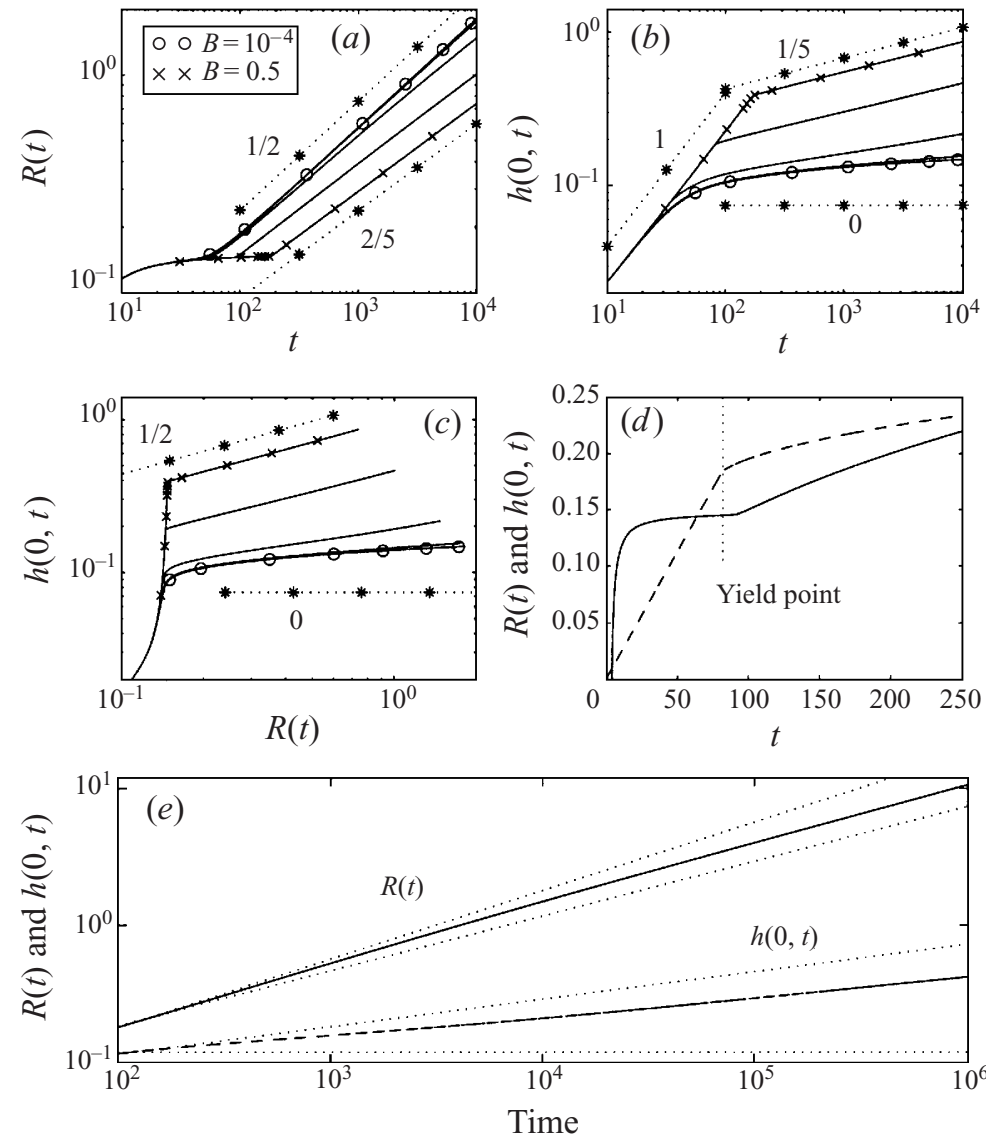

FIGURE 8. The variation in the radius and height with time for various values of $B\left(10^{-4}, 10^{-3}, 10^{-2}\right.$, 0.1 and 0.5. (a) $R(t)$ and $(b) h(0, t)$. (c) $h(0, t)$ plotted against $R(t)$ and $(d)$ the early development for the particular case $B=0.1$; the solid line is $R(t)$ and the dashed line is $h(0, t)$. Characteristic scalings for the Newtonian and yield-stress-dominated cases are also shown by the dotted lines and stars. (e) $R(t)$ (solid line) and $h(0, t)$ (dashed line) over a longer timescale; the dotted lines again show the viscous and yield-stress-dominated scalings. $\mu=10^{-2} B$.

yield-stress-dominated dynamics at a time

$$
t_{B}=\frac{q^{1 / 4}}{B^{2}}
$$

in dimensionless units, where $q=\pi r_{*}^{4} w_{0} / 2$ is the dimensionless effusion rate. For example, $t_{B}=944$ for $B=0.01$, but no transition occurs for such times in figure $8(e)$. The dimensional analysis assumes a switch between dominant behaviours but, evidently, the dome chooses a more balanced path between the two extremes.

\subsection{Variations with power-law index}

The clarity of this picture of the dynamics of lava-dome expansion is obscured when we allow $n$ to vary; once the fluid flows it now follows a nonlinear stress-strain-rate relation and the resulting dynamical changes can compete with or complement the effect of yield stress. Note that experiments invariably have $n<1$, corresponding to shear thinning; physically this models the destruction of the internal structure of the 


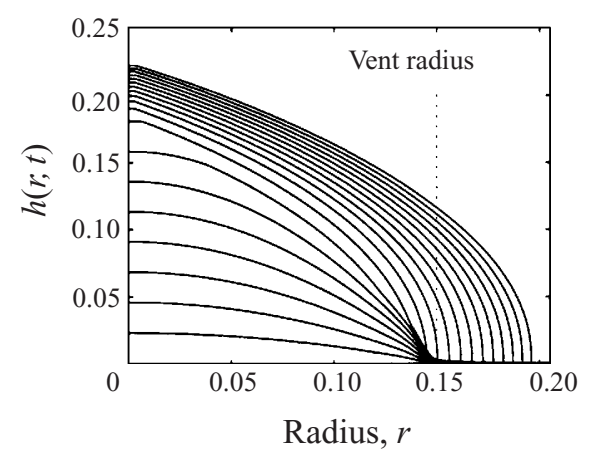

FIGURE 9. The initial evolution of the height field for $B=0.1$ and $\mu=10^{-4}$. Shown are snapshots of the solution every 25 time units.

fluid (such as brittle crystal structure or particulate interlinkage) by the increasing shear. Results for $n=1 / 2, n=1 / 3$ and $n=1 / 5$ are shown in figures 10-12.

Figure 10 shows the evolution of radius and height for fluids with different values for $B$ and $n$. The cases without yield stresses in $(a)$ and $(b)$ illustrate how reducing $n$ alters the $B=0$ scalings, and the evolution of radius and height follows temporal dependences that scale more closely with the yield-stress-dominated limit. The reason why this is the case is seen immediately from the scaling exponents in (4.6); for small $n$, these approach the values $1 / 5$ and $2 / 5$, respectively. As can be seen from figures 11 and 12, this feature leads to domes that are significantly less influenced by the introduction of the yield stress.

In other words, the power-law behaviour of the fluid can masquerade as a yieldstress effect. This has potentially important ramifications for studies that attempt to identify yield stress values from the morphology of flowing lava (Shaw 1969; Walker 1973; Hulme 1974; Blake 1990). Yield stress estimates derived from studies of static fluid do not, of course, suffer this problem (Fink \& Griffiths 1998).

This problem is also commonly encountered in chemical engineering: rheometers that operate with a controlled strain rate are not suitable to distinguish between yield stress effects and power-law behaviour because the differences are only evident at low shear rates where devices are prone to experimental error. A proper discrimination requires a controlled stress rheometer. But our model of an expanding lava dome is closer to having a controlled strain rate because the source represents a fixed material influx. Nevertheless, if we allowed the source to become time-dependent and, in particular, varied the inflow rate with the basal shear stress, we might move towards a situation more like the controlled stress rheometer. Then the morphology of the dome expansion might better distinguish the two effects.

\section{Basal sliding}

As a final piece of the isothermal theory of dome expansion, we relax the condition of no slip on the base of the fluid and allow the expanding dome to slide. In viscoplastic fluids, many experiments show significant slip near bounding walls as a result of the migration of particles, crystals or polymers away from regions of high shear. This migration leaves behind a layer of relatively dilute and less viscous fluid that effectively lubricates the wall (Barnes 1995).

This type of extension of the theory may also be necessary when modelling extrusions below water, where the plate may have a reduced coefficient of friction 

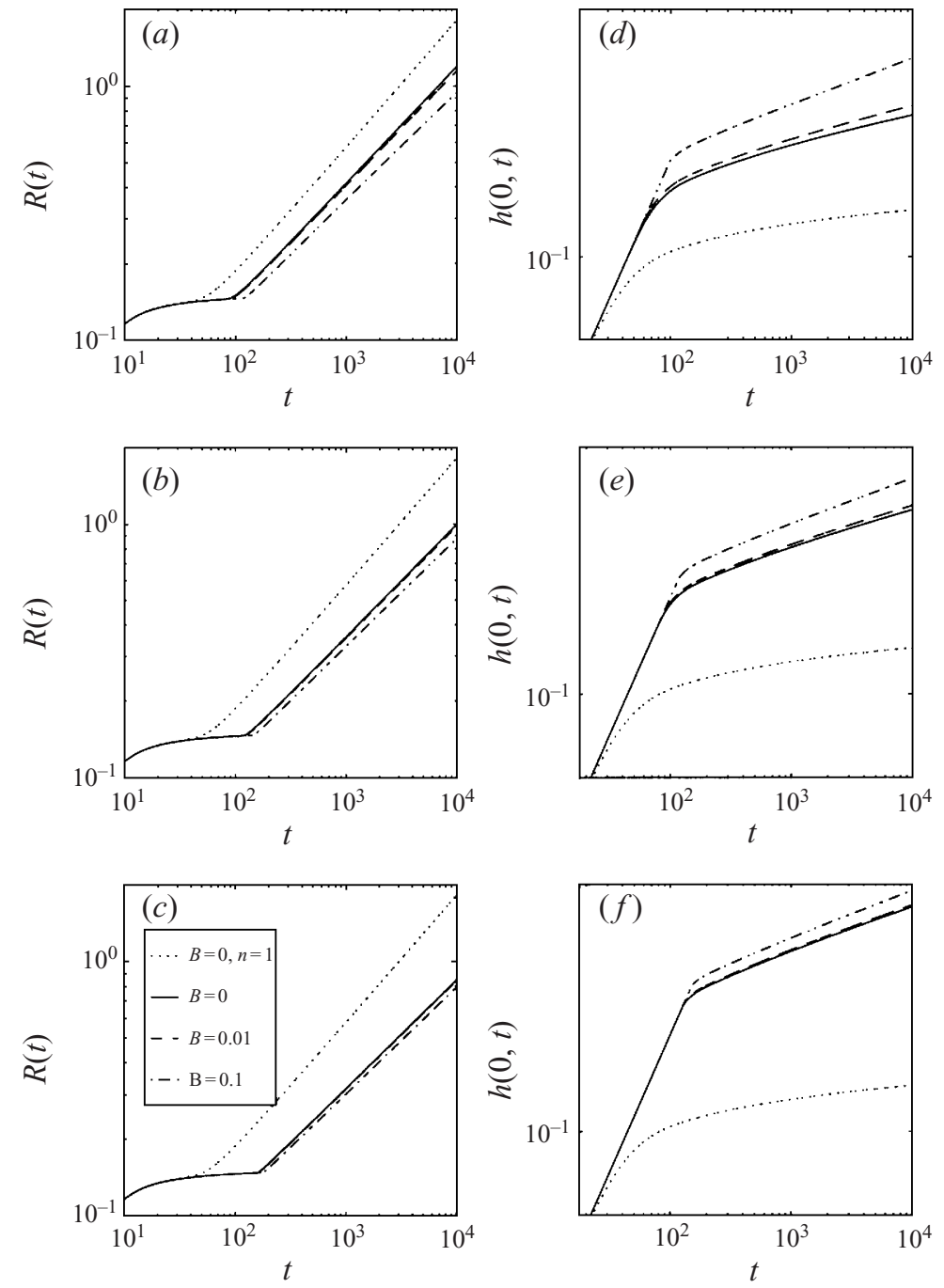

FIGURE 10. Evolution of radius and dome height for various values of $B$ and $n:(a, d) n=1 / 2$, $(b, e) n=1 / 3,(c, f) n=1 / 5 . \mu=10^{-2} B$.

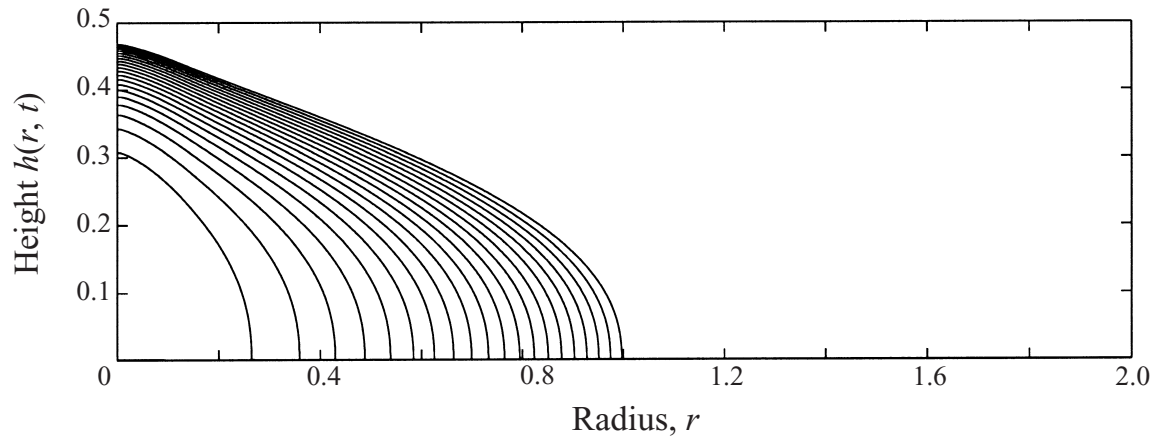

Figure 11. Evolution for $B=0$ and $n=1 / 3$. Shown is the height field, $h(r, t)$, every 500 time units. 


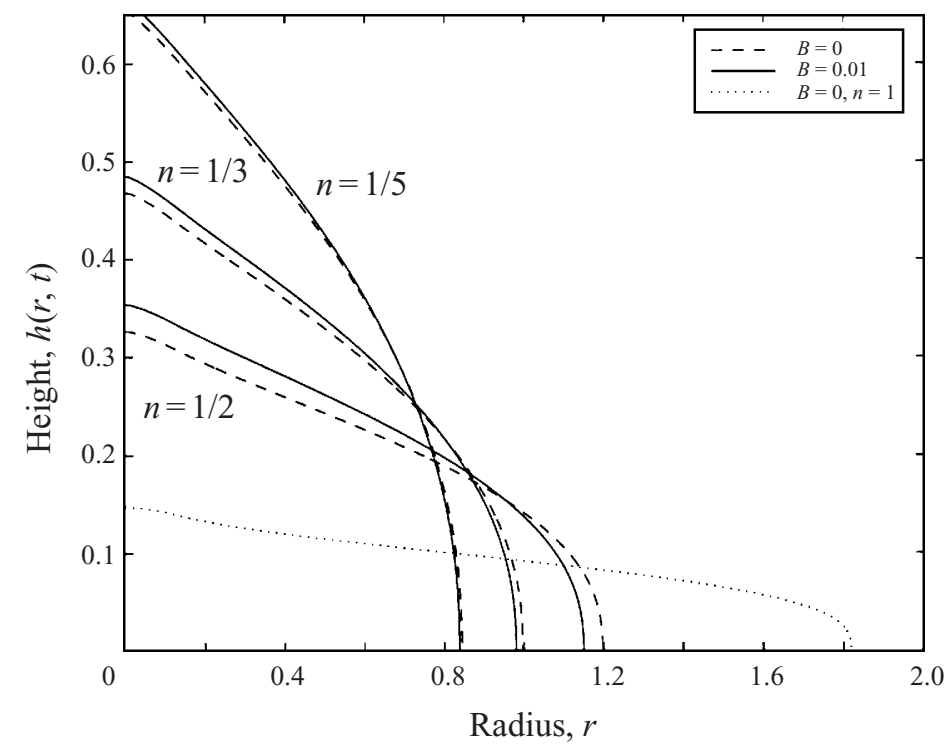

FIGURE 12. Dome shapes for varying $B$ and $n$ after $t=10^{4} \cdot \mu=10^{-2} B$.

(Griffiths \& Fink 1997); such experiments are typical in non-isothermal studies (Hallworth et al. 1987). Moreover, in real lavas (and in glaciers), motion over a rough base can take place through a succession of solidification and melting. This process can produce an effective slip (Hutter 1983).

In many engineering contexts, the no-slip condition is replaced by some empirical law relating the stress at the wall to the sliding velocity. Here we adopt the relation,

$$
\begin{array}{rlrl}
\left|\tau_{r z}\right| & =B_{w}+p_{0} \Omega\left(p_{0}\right) u_{b}^{\alpha} & & \text { if }\left|\tau_{r z}\right|>B_{w}, \\
u_{b}=0 & & \text { if }\left|\tau_{r z}\right|<B_{w}
\end{array}
$$

in dimensionless notation, where $p_{0}=p(r, z=0, t)$ is the pressure at the base of the fluid, $u_{b}$ is the radial sliding speed, and $B_{w}=\tau_{w} H / \eta U$ is a Bingham number that measures a yield stress at the base (so the fluid only slips if the stress exceeds a certain threshold, $\left.\tau_{w}\right)$. Also, $\alpha$ is some power-law index and the arbitrary function $\Omega\left(p_{0}\right)$ allows for a nonlinear friction law. Equation (7.1) is a version of Mooney's law (Mooney 1931). Without the basal yield stress, boundary conditions of this kind are used in glaciology (Hutter 1983; Fowler 1989; Morland 1997) and for flows of concentrated suspensions (Benbow \& Bridgwater 1993).

We may now proceed with the asymptotic expansion once again. The calculation is little different; manipulations like those used earlier lead to

$$
\begin{aligned}
U(r, t) & =\int_{0}^{h}(h-z) u_{z}(r, z, t) \mathrm{d} z \\
& =-\frac{n}{n+1}\left|h_{r}\right|^{1 / n-1} Y^{1+1 / n}\left(h-\frac{n Y}{2 n+1}\right) h_{r} \vartheta(Y) .
\end{aligned}
$$

However, $U(r, t)$ is no longer the vertical average of the radial outflow speed. Rather,

$$
U(r, t)=\int_{0}^{h} u(r, z, t)-h u_{b} .
$$


Moreover, from our sliding law,

$$
u_{b}=\left[\frac{-h_{r} Y_{w}}{h \Omega(h)}\right]^{1 / \alpha},
$$

where

$$
Y_{w}=\left(h-\frac{B_{w}}{\left|h_{r}\right|}\right)_{+} .
$$

This minor modification introduces a new term into the evolution equation, which is now

$$
h_{t}+\frac{1}{r} \partial_{r}\left[r\left(U+h u_{b}\right)\right]=w_{s} .
$$

Note that, if $B_{w}<B$ (for pastes $B_{w}$ is typically $10-30 \%$ of $B$ ), the fluid layer can be insufficiently stressed to shear internally, but it may still slide if $Y_{w}>0$. Again, there is a conceptual difficulty with this interpretation of the radial expansion: the fluid cannot be truly unyielding, but is order $\epsilon$ above the yield stress.

We illustrate the effects of sliding on the character of the dome expansion by choosing $\Omega(h)=$ constant, $\alpha=1$ and $B_{w}=0$. Then $u_{b}=-h_{r} / \Omega$. Some numerical results for this case are illustrated in figure 13 .

Evidently, the slip of the fluid breaks the characteristic temporal scalings of radius and height for the yield-stress-dominated limit, which would otherwise be found for the particular case shown in figure $13(B=0.1)$. Instead, there is no longer a similarity scaling for the slipping dome. Note that sliding also changes the shape of the dome near its edge, and, in particular, produces a characteristic 'skirt'. This feature might be useful in empirically estimating the degree of sliding simply from the dome shape.

In fact, a difference in the temporal scalings of radius and height was previously noted by Griffiths \& Fink (1997) between isothermal extrusions in air and below water. They found that domes below water were significantly lower than dry domes, a feature that is naturally explained in terms of basal slip. However, the experimental domes also showed little tendency to form a leading 'skirt'. Perhaps the fracturing process mentioned by Griffiths \& Fink offers a better explanation of the differing experimental scalings than basal slip.

\section{Experimental results}

To provide a laboratory comparison with the theoretical results we conducted experiments with kaolin-water slurry; these are similar to Hulme (1974) and Blake (1990). In particular, we extruded slurries in the fashion illustrated in figure 2. We performed two sets of experiments with two quite different experimental configurations: one is described by Shen (1998), and the results of a particular experiment with the other set-up are reported here (the extrusions showed no essential differences in the two cases).

\subsection{Restoring the dimensions}

To compare theory and observation, we must first restore the dimensions in our numerical solutions. To do this we need to estimate the length scales, $L$ and $H$, and the characteristic velocity, $V$.

To fix the horizontal length scale we match the dimensional vent radius, $L r_{*} \equiv$ $0.15 \mathrm{~L}$, with the size of the opening in the experimental apparatus: $R_{*} \approx 1.5 \mathrm{~mm}$. Thus $L=1 \mathrm{~cm}$. 

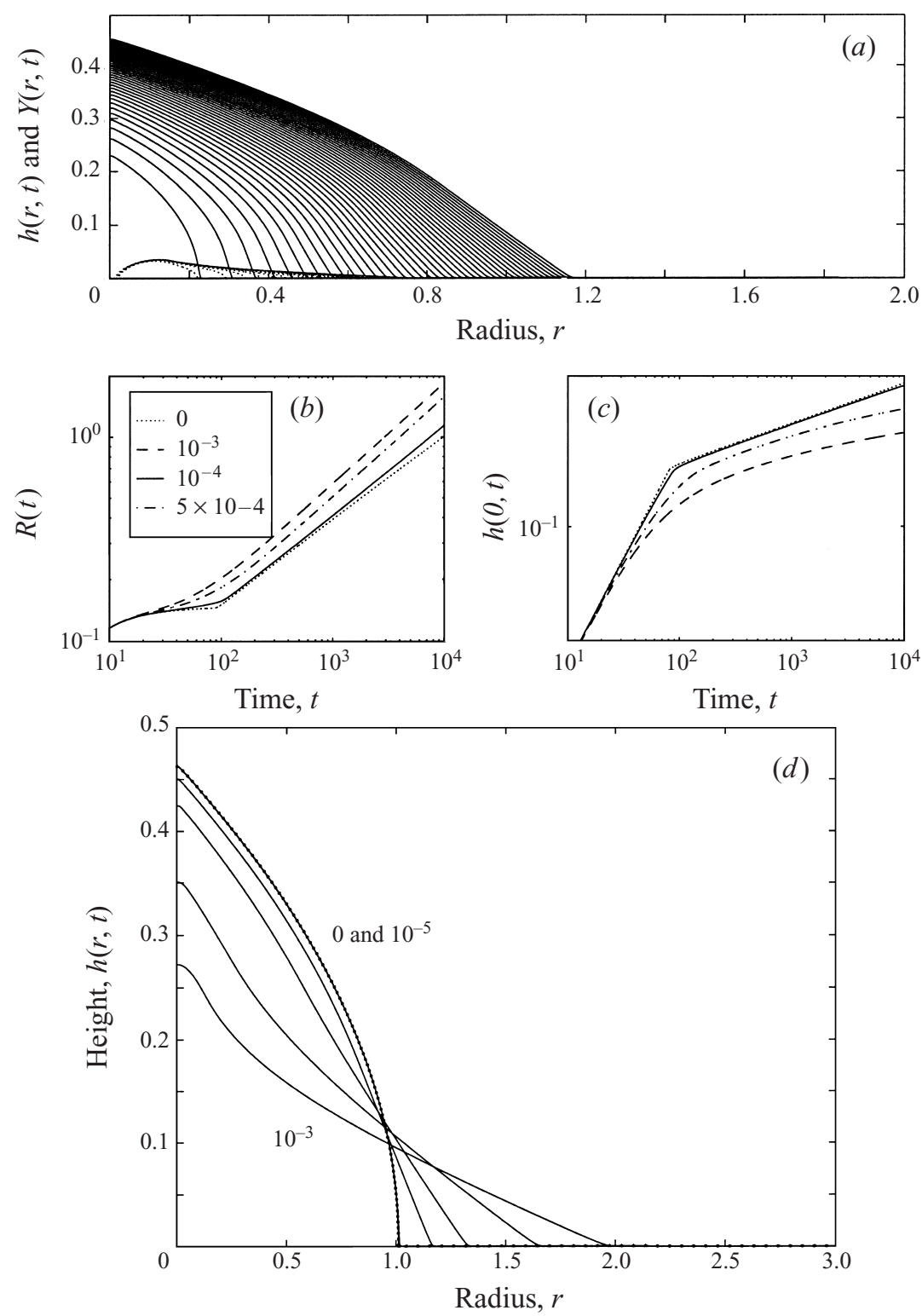

FiguRE 13. Evolution for a dome with basal sliding. $B=0.1$ and $\mu=10^{-4}$. (a) The height field every 500 time units for $\Omega=10^{4}$. (b,c) The evolution of the dome radius and height for models with $\Omega^{-1}=10^{-3}, 5 \times 10^{-4}, 10^{-4}$ and $0 .(d)$ Dome shapes at $t=10^{4}$ for $\Omega^{-1}=10^{-3}, 5 \times 10^{-4}, 2 \times 10^{-4}$, $10^{-4}, 10^{-5}$, and 0

We also have the dimensionless flow rate (obtained by integrating the source term over the vent),

$$
q=\frac{1}{2} \pi r_{*}^{4} w_{0},
$$

in which we select $w_{0}=0.1$, as in $\S 7$. In dimensional units, the volume flux is

$$
Q=L H V q .
$$


But we also have the relation

$$
V=\frac{\rho g H^{3}}{\eta L} \quad \text { or } \quad V^{n}=\frac{\rho g H^{2+n}}{K L},
$$

since $\eta=K V^{n-1} / H^{n-1}$. Thus, on eliminating $V$ :

$$
H=\left(\frac{K L^{1-n} Q^{n}}{\rho g q^{n}}\right)^{1 /(2 n+2)} .
$$

This allows us to compute $H$ and $V$ given $Q$ and $L$. Hence we may reconstruct the dimensional radius, height and time. Finally we estimate $B$ :

$$
B=\frac{\tau_{p} H}{\eta V} \equiv \tau_{p}\left(\frac{L^{2 n} q^{n}}{\rho^{n} g^{n} K Q^{n}}\right)^{1 /(n+1)} .
$$

The definitions above amount to evaluating $\epsilon=H / L$ and $B$ in terms of the experimental parameters. In fact, we could have based our non-dimensionalization of $\S 2.3$ on these parameters, and couched the formalism accordingly. One consequence of our current non-dimensionalization is that the velocity scale is given by balancing the viscous shear stress against the hydrostatic pressure gradient. But in yield-stressdominated fluids, it would be preferable to balance that gradient against the yield stress.

Also, in our numerical experiments, $q=\pi r_{*}^{4} w_{0} / 2 \sim 10^{-4}$. It is this relatively small non-dimensional effusion rate that leads to the deceptively low value of $B$ at which we enter the yield-stress-dominated regime.

Nevertheless, $\S 2.3$ follows the standard routes of lubrication analysis in Newtonian and non-Newtonian fluid mechanics, and for this reason we have elected to delay the reformulation in terms of the experimental parameters until now.

\subsection{Comparison of theory and experiment}

We compare theory and experiment in figure 14. The particular experiment is conducted with a slurry of density $\rho=1.32 \mathrm{~g} \mathrm{~cm}^{-3}$, and for which we fit the rheology with a Herschel-Bulkley model with parameters, $\tau_{p}=14 \mathrm{~Pa}, K=15(\mathrm{Pas})^{n}$, and $n=0.35$. The flow rate is $8.445 \times 10^{-2} \mathrm{~cm}^{3} \mathrm{~s}^{-1}$.

With the relevant dimensional scalings, we calculate the Bingham number as $B=0.0875$. This indicates that the dome is close to the yield-stress-dominated limit. However, as also indicated in the figure, there is certainly some difference between the predictions of the thin-layer equation and Nye's asymptotic solution given in $\S 4$.2. In any case, theory and experiment show reassuring agreement.

\section{Conclusion}

In this study we have presented a theory with which we can analyse isothermal lava flow. In particular, with the thin-film evolution equation, we may attempt to pick apart the physical ingredients in the problem. In this way we hope to separate effects such as viscosity, yield stress, shear thinning and basal sliding, and identify which could be most important in real lava domes. Within the limitations of the theory here, we identify the values of $B$ for which we enter the yield-stress-dominated limit; for real lava domes the values of $B$ are close to that regime suggesting that yield stresses are indeed important. 

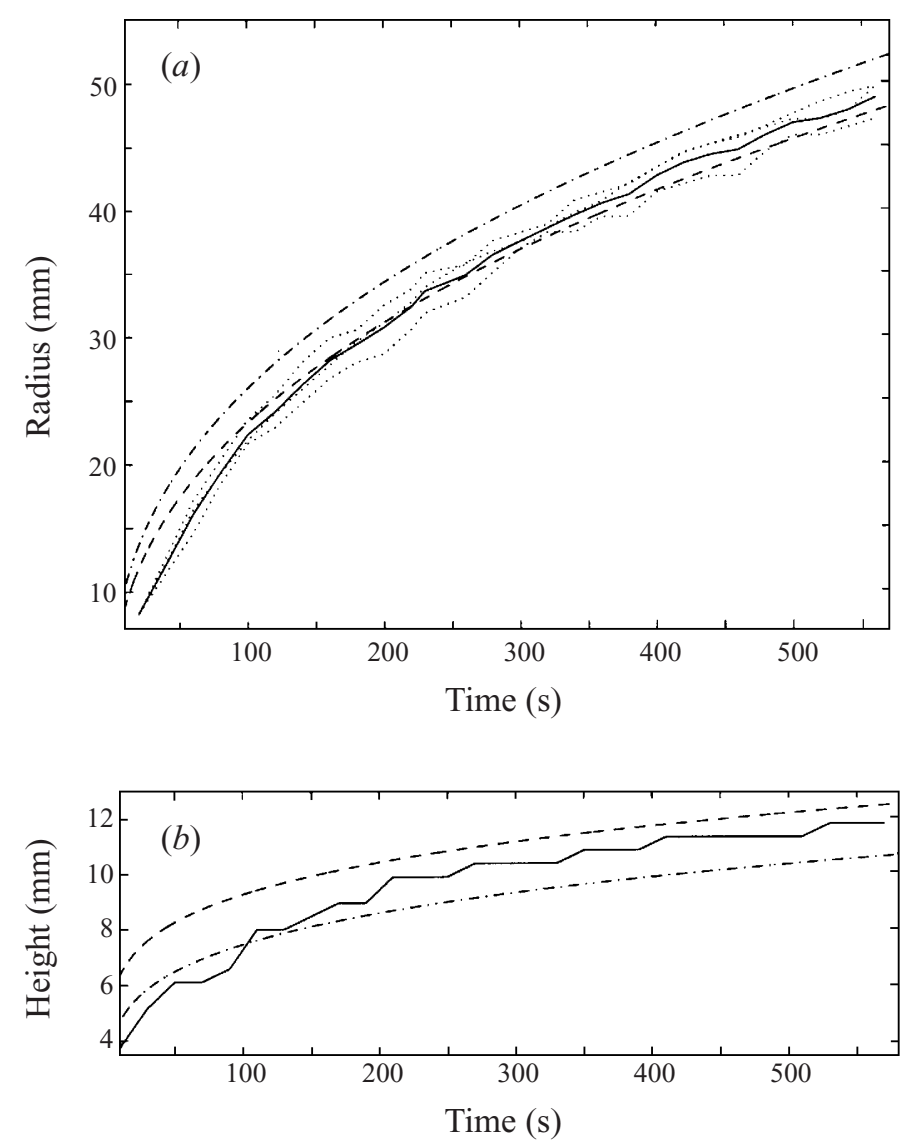

FIGURE 14. Experimental and theoretical comparison of dome evolution. (a) The radius: three measurements shown by dotted lines (part of the difference in the three curves is due to the fact that the dome was not precisely axisymmetrical, but showed slight asymmetry), that are averaged to compute the solid line. The dashed line is the analogous theoretical curve from solving the thin layer equation, and the dot-dashed line is that for Nye's asymptotic solution. (b) Comparison of height measurements, but there is only a single experimental curve (the solid line).

The results agree with the laboratory experiments that we have also conducted. This gives us extra motivation to continue on to the non-isothermal problem that we will describe in future work.

We should, however, warn the reader of some deficiencies in the thin-layer theory. One drawback of the asymptotic reduction of the problem has already appeared in the discussion of numerical results: the expansion must surely fail close to the centre of the dome, and the physical significance of solutions that appear to develop radial gradients near $r=0$ is questionable. A similar breakdown of the asymptotic theory arises where $h \rightarrow 0$. We have tacitly avoided a discussion of this contact ring here by taking initial conditions in which fluid was present everywhere. But we could explicitly consider the contact ring in more detail, much as has been done in a variety of studies of spreading of liquid drops (Greenspan 1978; Ehrhard \& Davis 1991; Ehrhard 1993). This entails a more involved discussion of the dynamics of the contact ring and draws in more physics. Notably, as the asymptotic expansion is currently set out, the theory 
must break down in order for the dome to expand: the no-slip condition rules out expansion without some kind of local overturning of the fluid surface at the dome edge. It is typical to avoid this inconsistency by allowing for some slip of the contact ring (Dussan V. 1979); basically, the no-slip condition is relaxed using a slip law like that described in $\S 7$.

One might also argue that if the extrusion takes place on a surface that is not perfectly horizontal, then the dome will slump to one side, and we will lose the axisymmetry that underlies the theory. Indeed, Fink \& Griffiths (1992) and Coussot, Proust \& Ancey (1996) display experiments in which just this effect was studied experimentally. However, whereas many geophysical settings for lava domes will not be horizontal, there are many examples of domes that are nearly axisymmetric, suggesting that the slumping is not important (see figure 1). Moreover, in the laboratory, we may engineer the plate to be as horizontal as desired. Thus we do not consider desymmetrizing effects to be especially important. Nevertheless, provided the slope is not too steep (the angle made between the plate and the horizontal is order $\epsilon$ or less), we can easily add the effects of the slope into the thin-layer theory; the equations become two-dimensional and the slope enters as a symmetry-breaking term. The extension of the theory follows that for flows on planes by Balmforth \& Craster (1999).

Finally, we mention that the numerical computations presented here have exclusively dealt with the case of a constant source. It is largely as a result of this prescription that we have difficulty in distinguishing power-law behaviour from the yield stress. However, we need not prescribe $w_{0}(t)$ in this way, and there may be better source functions that facilitate a discrimination of the two effects. Such modifications to our model may guide future experiments.

But there are also geophysical motivations for allowing the source to be timedependent. For example, the lava flow probably begins as the upcoming fluid forces its way through the overlying rocks and gradually opens a vent. Thus, initially, the flow rate slowly increases. Moreover, extrusions may be of limited duration if the outflowing magma is of finite volume, or if the vent ultimately closes due to the reduction in subterranean pressure caused by the outflow and the solidification of the lava. The pressure of the expanding dome may also react back on the fluid moving up the vent, leading to a dynamical control of the source (Stasiuk \& Jaupart 1997). In other words, the mechanics of the source can be a complicated, time-dependent process. Indeed, there is geophysical evidence that dome growth can take place through a sequence of distinct extrusions rather than a long continuous affair (for example, in the case of Mount St. Helens, it is estimated that about fifteen distinct episodes created the dome; Swanson \& Holcomb 1989). But whatever is needed for the additional physical input to the problem, the theory can be easily extended by simply modifying the source function, $w_{s}(r, t)$; our evolution equation remains a concise description of the expanding dome.

We thank both R. Griffiths and J. Fink for constructive criticisms of the manuscript, and a referee for drawing our attention to some useful references. The financial support of an EPSRC Advanced Fellowship is gratefully acknowledged by R. V.C. This work was done partly during the 1998 Geophysical Fluid Dynamics summer study program, Woods Hole Oceanographic Institution. N. J. B. was partially supported by the NSF Grant OCE-9616017 and an EPSRC Visiting Fellowship Grant GR/M50409, and thanks the ISI Foundation, Torino, and Imperial College, London, for hospitality during part of this work. 


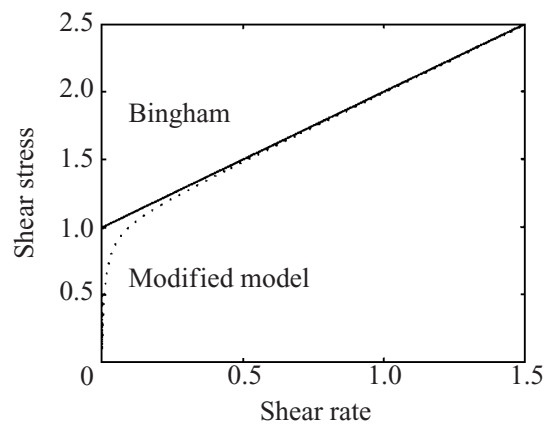

FIGURE 15. Constitutive models for the Bingham fluid and its regularization according to (A 1); the scales are arbitrary in this figure.

\section{Appendix. Regularization}

Numerical investigations utilizing the Bingham and Herschel-Bulkley models are complicated by the presence of the discontinuity in the derivative of the stressstrain-rate relation (there is no obstacle to careful analytical study). There are several avenues that can ease the numerical simulations. We adopt a route that has some physical basis, and regularize the constitutive model by, in effect, smoothing out the discontinuity. In the non-dimensional units of the main text, we regularize by adopting the new constitutive law,

$$
\tau_{i j}=\left(|\dot{\gamma}|^{n-1}+\frac{B}{\sqrt{\mu^{2}+\dot{\gamma}^{2}}}\right) \dot{\gamma} .
$$

The parameter $\mu$ modifies the constitutive model as indicated in figure 15; the fluid becomes weakly yielding at low strain rates, as is suggested by experiments with many real non-Newtonian materials (Barnes \& Walters 1985). There are many possible forms for the modified model; equation (A 1) has no special significance among all these possibilities. Provided $\mu$ is small, the form of the modification is not important; we use (A 1) essentially only as a numerical device that ultimately smooths out the function $U(r, t)$ of the main discussion. We describe this in detail for the Bingham fluid, and generalize the formulae to general $n$ at the end of this Appendix.

In the thin-layer theory, the leading-order non-dimensional equations are summarized as

$$
p_{r}=\partial_{z} \tau_{r z} \quad \text { and } \quad p_{z}=-1 .
$$

With the stress-free boundary condition, these may be integrated to

$$
p=h-z \quad \text { and } \quad \tau_{r z}=-h_{r}(h-z) .
$$

The constitutive model, on the other hand, leads to

$$
\tau_{r z}=u_{z}\left(1+\frac{B}{\sqrt{\mu^{2}+u_{z}^{2}}}\right),
$$

for $n=1$. This is a quartic equation for $u_{z}$. To generate the evolution equation we need to solve (A 4), and then integrate the product of the result with $h-z$ in order to provide the vertical integral of $u$. We will not, however, do this explicitly.

For a flow of uniform depth some solutions of the quartic are displayed in 

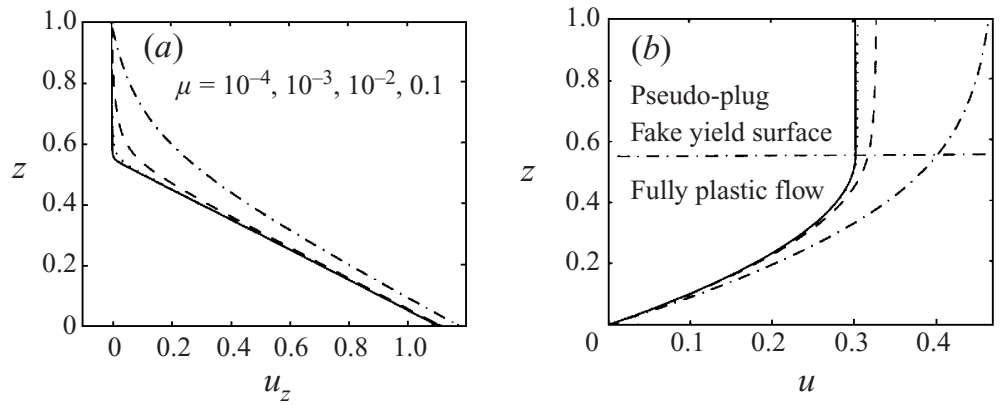

FigURE 16. Sketches of the vertical profiles of $(a)$ the velocity gradient, $u_{z}$, and $(b) u$ for different values of $\mu$.

figure 16. Importantly, when $\mu$ is small, the profile develops the distinctive character of the Bingham model - a fully plastic flow below a pseudo-plug.

Rather than deal with the cumbersome formulae that result from solving the quartic, we use a simpler regularization scheme based on the limits of (A 4) for $\tau_{r z}-B \gg \mu$ and $B-\tau_{r z} \gg \mu$. In the first limit, the stresses exceed the yield values and $\left|u_{z}\right| \gg \mu$. Hence,

$$
u_{z} \sim \tau_{r z}-B
$$

This gives the usual Bingham result for the velocity profile in the fully plastic region. Moreover, the contribution to the vertical integral of $u$ is given by

$$
\int_{0}^{Y_{-}}(h-z) u_{z} \mathrm{~d} z+O(\mu),
$$

where $Y_{-}$is $O(\mu)$ below the fake yield surface, $z=Y$, as defined in the main text.

In the other limit, we have the plug flow with

$$
u_{z} \sim \frac{\mu \tau_{r z}}{B} .
$$

Hence the fluid moves in a very viscous Newtonian fashion. The contribution of the pseudo-plug to the vertical integral of $u$ is expressible as

$$
\int_{Y_{+}}^{h}(h-z) u_{z} \mathrm{~d} z,
$$

where $Y_{+}$is $O(\mu)$ above the fake yield surface. This contribution is of order $\mu$, and is significantly smaller than (A 6). In addition there is another contribution from the vicinity of the yield surface, but this again is small. Hence, provided $Y \gg \mu$, the evolution equation remains unchanged.

The difficulty, however, is when we encounter a situation in which the plug flow expands to encompass the entire fluid layer. In that circumstance, (A 7) holds throughout the vertical section and consequently,

$$
U(r, t) \equiv \int_{0}^{h} u \mathrm{~d} z \sim-\frac{1}{3 B} \mu h^{3} h_{r} .
$$

Thus to regularize our model we look for a replacement of the formula (3.15) for $U$ 

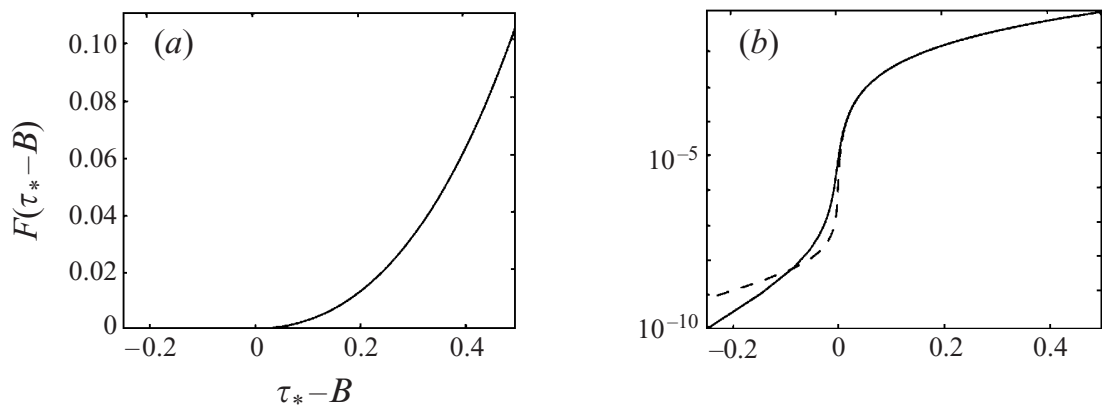

FIGURE 17. Comparison of exact and approximate regularizations for $\mu=10^{-4}$ and $B=0.5$. (a) $\int_{0}^{\tau_{*}} \dot{\gamma} \tau \mathrm{d} \tau$, where $\tau_{*}=-h h_{r}$, and the result in (A 10), divided by $h_{r}^{2}$, against $\tau_{*}-B$. In $(b)$ any differences are amplified by using logarithmic scalings. The dashed line is the exact result (numerically integrated), and the solid curve is our approximation. The errors for $\tau_{*}<B$ are inconsequential since the vertical integral of $u$ is very small there.

which limits to a small, Newtonian-like form such as (A9). In particular, we select

$$
U(r, t) \approx-\frac{1}{24}(3 h-Y)\left[Y+\sqrt{Y^{2}+\frac{\mu h^{3 / 2}}{B\left|h_{r}\right|^{1 / 2}}}\right]^{2} h_{r} .
$$

This formula does not limit quite to (A 9) when $Y$ is negative, but it compares well with the exact answer over the range of interest in $h$ and $h_{r}$, as illustrated in figure 17. The comparison is made by plotting $\int_{0}^{\tau_{*}} \dot{\gamma} \tau \mathrm{d} \tau$, where $\tau_{*}=-h h_{r}$, and the result in (A 10), multiplied by $h_{r}^{2}$ (both quantities can be expressed as functions of $\tau_{*}-B$ ).

The selection (A 10) does not completely solve all our problems with the evolution equation. Another difficulty emerges because $\left|h_{r}\right|$ appears both explicitly and in $Y$. To avoid this second non-smooth function we again turn to the constitutive model (A 1). The difficulty in this instance arises where $h_{r}$ changes sign. Near those locations, $\tau_{r z}$ is small. We rearrange (A 1) into

$$
\dot{\gamma}=-h_{r}\left[h-z+\frac{B}{\sqrt{h_{r}^{2}\left(1+\mu^{2} / \dot{\gamma}^{2}\right)}}\right] .
$$

But $\dot{\gamma} \sim-h_{r}(Y-z)$, and so a rough approximation near $h_{r}=0$ is furnished if we replace $\dot{\gamma}$ on the right-hand side of (A11) by $-\alpha h_{r}$, where $\alpha$ is given by some characteristic vertical average. Hence,

$$
\dot{\gamma} \approx-h_{r}\left[h-z+\frac{B}{\sqrt{h_{r}^{2}+\mu^{2} / \alpha^{2}}}\right] .
$$

This suggests that a plausible regularization of $\left|h_{r}\right|$ is $\sqrt{h_{r}^{2}+\mu^{2} / \alpha^{2}}$. In practice, we take $\alpha=1$.

We summarize the arguments of this Appendix by quoting the analogous regularization for the Herschel-Bulkley model:

$$
\begin{aligned}
U(r, t)=-\frac{n}{n+1}\left(h-\frac{n Y}{2 n+1}\right)\left\{\frac { 1 } { 2 } \left[Y+\sqrt{Y^{2}+}\right.\right. & \left.\left.\frac{\mu h^{3 n /(n+1)}}{B\left(h_{r}^{2}+\mu^{2}\right)^{1 / 4}}\right]\right\}^{1+1 / n} \\
& \times\left(h_{r}^{2}+\mu^{2}\right)^{(1-n) / 2 n} h_{r} .
\end{aligned}
$$


This formula smooths out all the problematic terms in the evolution equation; we need only take $\mu \ll B$ (and verify that the results are not dependent on the details of the regularization, which we indeed find to be the case).

\section{REFERENCES}

Balmforth, N. J. \& Craster, R. V. 1999 A consistent thin-layer theory for Bingham fluids. J. Non-Newtonian Fluid Mech. 84, 65-81.

Barenblatt, G. I. 1979 Similarity, Self-Similarity, and Intermediate Asymptotics. New York: Consultants Bureau.

BARNES, H. A. 1995 A review of the slip (wall depletion) of polymer solutions, emulsions and particle slip in viscometers - its cause, character, and cure. J. Non-Newtonian Fluid Mech. 56, 221-251.

Barnes, H. A. \& Walters, K. 1985 The yield stress myth? Rheol. Acta 24, 323-326.

Benbow, J. J. \& Bridgwater, J. 1993 Paste Flow and Extrusion. Clarendon.

BlaKe, S. 1990 Viscoplastic models of lava domes. In Lava Flows and Domes: Emplacement Mechanisms and Hazard Implications (ed. J. H. Fink), pp. 88-128. IAVCEI Proc. Volcanology, vol. 2. Springer.

Blom, J. G. \& Zegeling, P. A. 1994 Algorithm 731: a moving-grid interface for systems of onedimensional time-dependent partial differential equations. ACM Trans. Math. Software 20 194-214.

Burbidge, A. S. \& Bridgwater, J. 1999 Unsteady state planar divergent flow of extrusion pastes. Powder Technology (to appear).

Coussot, P. 1994 Steady laminar flow of concentrated mud suspensions in open channels. J. Hydr. Res. 32, 535-550.

Coussot, P. 1997 Mudflow Rheology and Dynamics. IAHR Monograph Series, Balkema.

Coussot, P., Proust, S. \& Ancey, C. 1996 Rheological interpretation of deposits of yield stress fluids. J. Non-Newtonian Fluid Mech. 66, 55-70.

Davies, T. R. H. 1986 Large debris flows: a macro-viscous phenomenon. Acta Mechanica 63, 161-178.

Dent, J. P. \& Lang, T. E. 1983 A biviscous modified Bingham model of snow avalanche motion. Ann. Glaciology 4, 42-46.

Dussan V., E. B. 1979 On the spreading of liquids on solid surfaces: static and dynamic contact lines. Ann. Rev. Fluid Mech. 11, 371-400.

EHrhaRD, P. 1993 Experiments on isothermal and non-isothermal spreading. J. Fluid Mech. 257, 463-483.

Ehrhard, P. \& DAVIS, S. H. 1991 Non-isothermal spreading of liquid drops on horizontal plates. J. Fluid Mech. 220, 365-388.

FinK, J. H. \& GRIFFITHS, R. W. 1992 A laboratory study of the surface morphology of lava flows extruded from point and line sources. J. Volcan. Geotherm. Res. 54, 19-32.

Fink, J. H. \& GRIFfiths, R. W. 1998 Morphology, eruption rates and rheology of lava domes: insights from laboratory models. J. Geophys. Res. 103, 527-545.

Fowler, A. C. 1989 A mathematical analysis of glacier surges. SIAM J. Appl. Maths 49, 246-263.

Fowler, A. C. 1992 Modelling ice sheet dynamics. Geophys. Astrophys. Fluid Dyn. 63, 29-65.

Greenspan, H. P. 1978 On the motion of a small viscous droplet that wets a surface. J. Fluid Mech. 84, 125-143.

GRIFFITHS, R. W. \& Fink, J. H. 1993 Effects of surface cooling on the spreading of lava flows and domes. J. Fluid Mech. 252, 667-702.

Griffiths, R. W. \& FinK, J. H. 1997 Solidifying Bingham extrusions: A model for the growth of silicic lava domes. J. Fluid Mech. 347, 13-36.

Hallworth, M. A., Huppert, H. E. \& Sparks, R. S. J. 1987 A laboratory simulation of basaltic lava flows. Modern Geol. 11, 93-107.

Herschel, W. H. \& Bulkley, R. 1923 Uber die viskositat und Elastizitat von Solen. Am. Soc. Testing Mater. 26, 621-633.

Huang, X. \& Garcia, M. H. 1998 A Herschel-Bulkley model for mud flow down a slope. J. Fluid Mech. 374, 305-333. 
Hulme, G. 1974 The interpretation of lava flow morphology. Geophys. J. R. Astron. Soc. 39, 361-383.

HuPPERT, H. E. 1982 The propagation of two-dimensional and axisymmetric viscous gravity currents over a rigid horizontal surface. J. Fluid Mech. 121, 43-58.

Hutter, K. 1983 Theoretical Glaciology. D. Reidel.

Johnson, R. E. 1984 Power-law creep of a material being compressed between parallel plates: a singular perturbation problem. J. Engng Maths 18, 105-117.

Keast, P. \& Muir, P. H. 1991 Algorithm 688 EPDCOL-a more efficient PDECOL code. ACM Trans. Math. Software 17, 153-166.

LaWrence, C. J. \& CoRfield, G. M. 1998 Non-viscometric flow of viscoplastic materials: squeeze flow. In Dynamics of Complex Fluids (ed. M. J. Adams, R. A. Mashelkar, J. R. A. Pearson \& A. R. Rennie), pp. 379-393. Imperial College Press/The Royal Society.

Lipscomb, G. G. \& DenN, M. M. 1984 Flow of Bingham fluids in complex geometries. J. Non-Newtonian Fluid Mech. 14, 337-346.

LiU, K. F. \& MeI, C. C. 1989 Slow spreading of Bingham fluid on an inclined plane. J. Fluid Mech. 207, 505-529.

LiU, K. F. \& MeI, C. C. 1991 Approximate equations for the slow spreading of a thin sheet of Bingham plastic fluid. Phys. Fluids A 2, 30-36.

McBirney, A. R. \& Murase, T. 1984 Rheological properties of magmas. Ann. Rev. Earth Planet. Sci. 12, 337-357.

Mooney, M. 1931 Explicit formulas for slip and fluidity. J. Rheol. 2, 210-222.

Morland, L. W. 1997 Radially symmetric ice sheet flow. Phil. Trans. R. Soc. Lond. A 355, 1873-1904.

N'Guyen, Q. D. \& Boger, D. V. 1992 Measuring the flow properties of yield-stress fluids. Ann. Rev. Fluid Mech. 24, 47-88.

NYE, J. F. 1952 The mechanics of glacier flow. J. Glaciol. 2, 82-93.

OLdRoyd, J. G. 1947 Two-dimensional plastic flow of a Bingham solid: a plastic boundary-layer theory for slow motion. Proc. Camb. Phil. Soc. 43, 383-395.

Pinkerton, H. \& Stevenson, R. J. 1992 Methods of determining the rheological properties of magmas at sub-liquidus temperatures. J. Volcan. Geotherm. Res. 53, 47-66.

Shaw, H. R. 1969 Rheology of basalt in the melting range. J. Petrol. 10, 510-535.

SHEN, A. 1998 Mathematical and analog modeling of lava dome growth. In Proc. 1998 GFD Summer School. Woods Hole Oceanographic Institution.

Smith, J. V. 1997 Shear thickening dilatancy in crystal-rich flows. J. Volcan. Geotherm. Res. 79, 1-8.

StASIUK, M. V. \& JAUPART, C. 1997 Lava flow shapes and dimensions as reflections of magma system conditions. J. Volcan. Geotherm. Res. 78, 31-50.

Stasiuk, M. V., Jaupart, C. \& Sparks, R. S. J. 1993 Influence of cooling on lava-flow dynamics. Geology 21, 335-338.

Swanson, D. A. \& Holcomb, R. T. 1989 Regularities in growth of the Mount St. Helens dacite dome, 1980-1986. In IAVCEI Proc. Volcanology, Vol.2, Lava Flows and Domes. Springer.

Walker, G. P. L. 1973 Lengths of lava flows. Phil. Trans. R. Soc. Lond. A 274, 107-118. 